\title{
A half-discrete Hardy-Hilbert-type inequality related to hyperbolic secant function
}

Bicheng Yang ${ }^{1 *}$ and Qiang Chen ${ }^{2}$

"Correspondence:
bcyang@gdei.edu.cn
${ }^{1}$ Department of Mathematics,
Guangdong University of
Education, Guangzhou, Guangdong
51003, P.R. China
Full list of author information is
available at the end of the article

available at the end of the article

\begin{abstract}
By applying weight functions and technique of real analysis, a half-discrete Hardy-Hilbert-type inequality related to the kernel of hyperbolic secant function and a best possible constant factor are given. The equivalent forms, the operator expressions with the norm, the reverses, and some particular cases are also considered.
\end{abstract}

MSC: 26D15; 47A07; 37A10

Keywords: Hardy-Hilbert-type inequality; weight function; equivalent form; reverse; operator

\section{Introduction}

If $p>1, \frac{1}{p}+\frac{1}{q}=1, f(x), g(y) \geq 0, f \in L^{p}\left(\mathbf{R}_{+}\right), g \in L^{q}\left(\mathbf{R}_{+}\right),\|f\|_{p}=\left(\int_{0}^{\infty} f^{p}(x) d x\right)^{\frac{1}{p}}>0$, and $\|g\|_{q}>0$, then we have the following Hardy-Hilbert integral inequality [1]:

$$
\int_{0}^{\infty} \int_{0}^{\infty} \frac{f(x) g(y)}{x+y} d x d y<\frac{\pi}{\sin (\pi / p)}\|f\|_{p}\|g\|_{q}
$$

where, the constant factor $\frac{\pi}{\sin (\pi / p)}$ is the best possible. If $a_{m}, b_{n} \geq 0, a=\left\{a_{m}\right\}_{m=1}^{\infty} \in l^{p}$, $b=\left\{b_{n}\right\}_{n=1}^{\infty} \in l^{q},\|a\|_{p}=\left(\sum_{m=1}^{\infty} a_{m}^{p}\right)^{\frac{1}{p}}>0$, and $\|b\|_{q}>0$, then we have the following HardyHilbert's inequality with the same best constant $\frac{\pi}{\sin (\pi / p)}[1]$ :

$$
\sum_{m=1}^{\infty} \sum_{n=1}^{\infty} \frac{a_{m} b_{n}}{m+n}<\frac{\pi}{\sin (\pi / p)}\|a\|_{p}\|b\|_{q}
$$

Inequalities (1) and (2) are important in analysis and its applications (see [1-5]).

Suppose that $\mu_{i}, v_{j}>0(i, j \in \mathbf{N}=\{1,2, \ldots\})$,

$$
U_{m}:=\sum_{i=1}^{m} \mu_{i}, \quad V_{n}:=\sum_{j=1}^{n} v_{j} \quad(m, n \in \mathbf{N}) .
$$

\section{Springer}


Then we have the following inequality ([1], Theorem 321):

$$
\sum_{m=1}^{\infty} \sum_{n=1}^{\infty} \frac{\mu_{m}^{1 / q} v_{n}^{1 / p} a_{m} b_{n}}{U_{m}+V_{n}}<\frac{\pi}{\sin (\pi / p)}\|a\|_{p}\|b\|_{q} .
$$

Replacing $\mu_{m}^{1 / q} a_{m}$ and $v_{n}^{1 / p} b_{n}$ by $a_{m}$ and $b_{n}$ in (4), respectively, we obtain an equivalent form of (4):

$$
\sum_{m=1}^{\infty} \sum_{n=1}^{\infty} \frac{a_{m} b_{n}}{U_{m}+V_{n}}<\frac{\pi}{\sin \left(\frac{\pi}{p}\right)}\left(\sum_{m=1}^{\infty} \frac{a_{m}^{p}}{\mu_{m}^{p-1}}\right)^{\frac{1}{p}}\left(\sum_{n=1}^{\infty} \frac{b_{n}^{q}}{v_{n}^{q-1}}\right)^{\frac{1}{q}} .
$$

For $\mu_{i}=v_{j}=1(i, j \in \mathbf{N})$, both (4) and (5) reduce to (2). We call (4) and (5) Hardy-Hilberttype inequalities.

Note The authors of [1] did not prove that (4) is valid with the best possible constant factor.

In 1998, by introducing an independent parameter $\lambda \in(0,1]$ Yang [6] gave an extension of (1) with the kernel $\frac{1}{(x+y)^{\lambda}}$ for $p=q=2$. Later, Yang [5] refined [6] by giving extensions of (1) and (2) as follows.

Assuming that $\lambda_{1}, \lambda_{2} \in \mathbf{R}, \lambda_{1}+\lambda_{2}=\lambda, k_{\lambda}(x, y)$ is a nonnegative homogeneous function of degree $-\lambda$ with $k\left(\lambda_{1}\right)=\int_{0}^{\infty} k_{\lambda}(t, 1) t^{\lambda_{1}-1} d t \in \mathbf{R}_{+}, \phi(x)=x^{p\left(1-\lambda_{1}\right)-1}, \psi(x)=x^{q\left(1-\lambda_{2}\right)-1}$, $f(x), g(y) \geq 0$,

$$
f \in L_{p, \phi}\left(\mathbf{R}_{+}\right)=\left\{f ;\|f\|_{p, \phi}:=\left\{\int_{0}^{\infty} \phi(x)|f(x)|^{p} d x\right\}^{\frac{1}{p}}<\infty\right\},
$$

$g \in L_{q, \psi}\left(\mathbf{R}_{+}\right),\|f\|_{p, \phi},\|g\|_{q, \psi}>0$, we have

$$
\int_{0}^{\infty} \int_{0}^{\infty} k_{\lambda}(x, y) f(x) g(y) d x d y<k\left(\lambda_{1}\right)\|f\|_{p, \phi}\|g\|_{q, \psi},
$$

where the constant factor $k\left(\lambda_{1}\right)$ is the best possible. Moreover, if $k_{\lambda}(x, y)$ keeps finite and $k_{\lambda}(x, y) x^{\lambda_{1}-1}\left(k_{\lambda}(x, y) y^{\lambda_{2}-1}\right)$ is decreasing with respect to $x>0(y>0)$, then for $a_{m}, b_{n} \geq 0$,

$$
a \in l_{p, \phi}=\left\{a ;\|a\|_{p, \phi}:=\left(\sum_{n=1}^{\infty} \phi(n)\left|a_{n}\right|^{p}\right)^{\frac{1}{p}}<\infty\right\},
$$

$b=\left\{b_{n}\right\}_{n=1}^{\infty} \in l_{q, \psi},\|a\|_{p, \phi},\|b\|_{q, \psi}>0$, we have

$$
\sum_{m=1}^{\infty} \sum_{n=1}^{\infty} k_{\lambda}(m, n) a_{m} b_{n}<k\left(\lambda_{1}\right)\|a\|_{p, \phi}\|b\|_{q, \psi}
$$

where the constant factor $k\left(\lambda_{1}\right)$ is still the best possible.

For $0<\lambda_{1}, \lambda_{2} \leq 1$ such that $\lambda_{1}+\lambda_{2}=\lambda$, we set

$$
k_{\lambda}(x, y)=\frac{1}{(x+y)^{\lambda}} \quad\left((x, y) \in \mathbf{R}_{+}^{2}\right) .
$$


Then by (7) we have

$$
\sum_{m=1}^{\infty} \sum_{n=1}^{\infty} \frac{a_{m} b_{n}}{(m+n)^{\lambda}}<B\left(\lambda_{1}, \lambda_{2}\right)\|a\|_{p, \phi}\|b\|_{q, \psi}
$$

where the constant $B\left(\lambda_{1}, \lambda_{2}\right)$ is the best possible, and

$$
B(u, v)=\int_{0}^{\infty} \frac{1}{(1+t)^{u+v}} t^{u-1} d t \quad(u, v>0)
$$

is the beta function. Clearly, for $\lambda=1, \lambda_{1}=\frac{1}{q}, \lambda_{2}=\frac{1}{p}$, inequality (8) reduces to (2).

In 2015, by adding some conditions, Yang [7] gave an extension of (8) and (5) as follows:

$$
\begin{aligned}
& \sum_{m=1}^{\infty} \sum_{n=1}^{\infty} \frac{a_{m} b_{n}}{\left(U_{m}+V_{n}\right)^{\lambda}} \\
& \quad<B\left(\lambda_{1}, \lambda_{2}\right)\left(\sum_{m=1}^{\infty} \frac{U_{m}^{p\left(1-\lambda_{1}\right)-1} a_{m}^{p}}{\mu_{m}^{p-1}}\right)^{\frac{1}{p}}\left(\sum_{n=1}^{\infty} \frac{V_{n}^{q\left(1-\lambda_{2}\right)-1} b_{n}^{q}}{v_{n}^{q-1}}\right)^{\frac{1}{q}},
\end{aligned}
$$

where the constant $B\left(\lambda_{1}, \lambda_{2}\right)$ is still the best possible.

Some other results including multidimensional Hilbert-type inequalities are provided by [8-25].

About the topic of half-discrete Hilbert-type inequalities with inhomogeneous kernels, Hardy et al. provided a few results in Theorem 351 of [1], but they did not prove that the constant factors are the best possible. However, Yang [26] gave a result with the kernel $\frac{1}{(1+n x)^{\lambda}}$ by introducing a variable and proved that the constant factor is the best possible. In 2011, Yang [27] gave the following half-discrete Hardy-Hilbert's inequality with the best possible constant factor $B\left(\lambda_{1}, \lambda_{2}\right)$ :

$$
\int_{0}^{\infty} f(x)\left[\sum_{n=1}^{\infty} \frac{a_{n}}{(x+n)^{\lambda}}\right] d x<B\left(\lambda_{1}, \lambda_{2}\right)\|f\|_{p, \phi}\|a\|_{q, \psi},
$$

where, $\lambda_{1}>0,0<\lambda_{2} \leq 1, \lambda_{1}+\lambda_{2}=\lambda$. Zhong and Yang [17,28-33] investigated several halfdiscrete Hilbert-type inequalities with particular kernels. Applying weight functions, a half-discrete Hilbert-type inequality with a general homogeneous kernel of degree $-\lambda \in \mathbf{R}$ with the best constant factor $k\left(\lambda_{1}\right)$ is obtained as follows:

$$
\int_{0}^{\infty} f(x) \sum_{n=1}^{\infty} k_{\lambda}(x, n) a_{n} d x<k\left(\lambda_{1}\right)\|f\|_{p, \phi}\|a\|_{q, \psi}
$$

which is an extension of (10) (cf. [34]). At the same time, a half-discrete Hilbert-type inequality with a general inhomogeneous kernel and the best constant factor is given by Yang [35]. In 2012-2014, Yang et al. published three books [36, 37] and [38] for building the theory of half-discrete Hilbert-type inequalities.

In this paper, by applying weight coefficients and technique of real analysis, a halfdiscrete Hardy-Hilbert-type inequality related to the kernel of hyperbolic secant function and the best possible constant factor is given, which is an extension of (11) for $\lambda=0$ and a particular kernel. The equivalent forms, the operator expressions with the norm, the reverses, and some particular cases are also considered. 


\section{Some lemmas}

In the following, we make appointment that $\mu_{i}, v_{j}>0(i, j \in \mathbf{N}), U_{m}$ and $V_{n}$ are defined by (3), $\mu(t)$ is a positive continuous function in $\mathbf{R}_{+}=(0, \infty)$,

$$
\begin{aligned}
& U(x):=\int_{0}^{x} \mu(t) d t<\infty \quad(x \in[0, \infty)), \\
& v(t):=v_{n}, t \in(n-1, n](n \in \mathbf{N}), \text { and } \\
& V(y):=\int_{0}^{y} v(t) d t \quad(y \in[0, \infty)), \\
& p \neq 0,1, \frac{1}{p}+\frac{1}{q}=1, \delta \in\{-1,1\}, f(x), a_{n} \geq 0\left(x \in \mathbf{R}_{+}, n \in \mathbf{N}\right),\|f\|_{p, \Phi_{\delta}}=\left(\int_{0}^{\infty} \Phi_{\delta}(x) f^{p}(x) d x\right)^{\frac{1}{p}}, \\
& \|a\|_{q, \Psi}=\left(\sum_{n=1}^{\infty} \Psi(n) b_{n}^{q}\right)^{\frac{1}{q}}, \text { where } \\
& \Phi_{\delta}(x):=\frac{U^{p(1-\delta \sigma)-1}(x)}{\mu^{p-1}(x)}, \quad \Psi(n):=\frac{V_{n}^{q(1-\sigma)-1}}{v_{n}^{q-1}} \quad\left(x \in \mathbf{R}_{+}, n \in \mathbf{N}\right) .
\end{aligned}
$$

Example 1 For $\rho, \gamma, \sigma>0, \alpha>-\rho$, $\sec h(u)=\frac{2}{e^{u}+e^{-u}}(u>0)$ is called the hyperbolic secant function (cf. [39]), we set $h(t)=\frac{\sec h\left(\rho t^{\prime}\right)}{e^{\alpha t^{\gamma}}}\left(t \in \mathbf{R}_{+}\right)$.

(i) Setting $u=\rho t^{\gamma}$, we find

$$
\begin{aligned}
k(\sigma) & :=\int_{0}^{\infty} \frac{\operatorname{sech}\left(\rho t^{\gamma}\right)}{e^{\alpha t^{\gamma}}} t^{\sigma-1} d t \\
& =\frac{1}{\gamma \rho^{\sigma / \gamma}} \int_{0}^{\infty} \frac{\sec h(u)}{e^{\frac{\alpha}{\rho} u} u^{\frac{\sigma}{\gamma}-1} d u} \\
& =\frac{2}{\gamma \rho^{\sigma / \gamma}} \int_{0}^{\infty} \frac{e^{-\frac{\alpha}{\rho} u} u^{\frac{\sigma}{\gamma}-1}}{e^{u}+e^{-u}} d u \\
& =\frac{2}{\gamma \rho^{\sigma / \gamma}} \int_{0}^{\infty} \frac{e^{-\left(\frac{\alpha}{\rho}+1\right) u} u^{\frac{\sigma}{\gamma}-1}}{1+e^{-2 u}} d u \\
& =\frac{2}{\gamma \rho^{\sigma / \gamma}} \int_{0}^{\infty} \sum_{k=0}^{\infty}(-1)^{k} e^{-\left(2 k+\frac{\alpha}{\rho}+1\right) u} u^{\frac{\sigma}{\gamma}-1} d u \\
& =\frac{2}{\gamma \rho^{\sigma / \gamma}} \int_{0}^{\infty} \sum_{k=0}^{\infty}\left[e^{-\left(4 k+\frac{\alpha}{\rho}+1\right) u}-e^{-\left(4 k+2+\frac{\alpha}{\rho}+1\right) u}\right] u^{\frac{\sigma}{\gamma}-1} d u
\end{aligned}
$$

By the Lebesgue term-by-term theorem (see [39]), setting $v=\left(2 k+\frac{\alpha}{\rho}+1\right) u$, we have

$$
\begin{aligned}
k(\sigma) & =\int_{0}^{\infty} \frac{\sec h\left(\rho t^{\gamma}\right)}{e^{\alpha t^{\gamma}}} t^{\sigma-1} d t \\
& =\frac{2}{\gamma \rho^{\sigma / \gamma}} \sum_{k=0}^{\infty} \int_{0}^{\infty}\left[e^{-\left(4 k+\frac{\alpha}{\rho}+1\right) u}-e^{-\left(4 k+2+\frac{\alpha}{\rho}+1\right) u}\right] u^{\frac{\sigma}{\gamma}-1} d u \\
& =\frac{2}{\gamma \rho^{\sigma / \gamma}} \sum_{k=0}^{\infty}(-1)^{k} \int_{0}^{\infty} e^{-\left(2 k+\frac{\alpha}{\rho}+1\right) u} u^{\frac{\sigma}{\gamma}-1} d u \\
& =\frac{2}{\gamma \rho^{\sigma / \gamma}} \sum_{k=0}^{\infty} \frac{(-1)^{k}}{\left(2 k+\frac{\alpha}{\rho}+1\right)^{\sigma / \gamma}} \int_{0}^{\infty} e^{-v} v^{\frac{\sigma}{\gamma}-1} d \nu
\end{aligned}
$$




$$
\begin{aligned}
& =\frac{2 \Gamma\left(\frac{\sigma}{\gamma}\right)}{\gamma(2 \rho)^{\sigma / \gamma}} \sum_{k=0}^{\infty} \frac{(-1)^{k}}{\left(k+\frac{\alpha+\rho}{2 \rho}\right)^{\sigma / \gamma}} \\
& =\frac{2 \Gamma\left(\frac{\sigma}{\gamma}\right)}{\gamma(2 \rho)^{\sigma / \gamma}} \xi\left(\frac{\sigma}{\gamma}, \frac{\alpha+\rho}{2 \rho}\right) \in \mathbf{R}_{+},
\end{aligned}
$$

where $\xi(s, a):=\sum_{k=0}^{\infty} \frac{(-1)^{k}}{(k+a)^{s}}(s, a>0)$ and

$$
\Gamma(y):=\int_{0}^{\infty} e^{-v} v^{y-1} d v \quad(y>0)
$$

is the gamma function (see [40]).

In particular, for $\alpha=\rho>0$ and $\gamma=\sigma$, we have $h(t)=\frac{\sec h\left(\rho t^{\sigma}\right)}{e^{\rho t^{\sigma}}}$ and $k(\sigma)=\frac{\ln 2}{\sigma \rho}$; for $\alpha=0$ and $\gamma=\sigma$, we find $h(t)=\sec h\left(\rho t^{\sigma}\right)$ and $k(\sigma)=\frac{\pi}{2 \sigma \rho}$.

(ii) We have $\frac{1}{e^{u}+e^{-u}}>0$ and $\left(\frac{1}{e^{u}+e^{-u}}\right)^{\prime}=-\frac{e^{u}-e^{-u}}{\left(e^{u}+e^{-u}\right)^{2}}<0$ for $u>0$. If $g(u)>0$ and $g^{\prime}(u)<0$, then for $\gamma>0, g\left(\rho t^{\gamma}\right)>0, \frac{d}{d t} g\left(\rho t^{\gamma}\right)=\rho \gamma t^{\gamma-1} g^{\prime}\left(\rho t^{\gamma}\right)<0$; for $y \in(n-1, n), g(V(y))>0$, $\frac{d}{d y} g(V(y))=g^{\prime}(V(y)) v_{n}<0(n \in \mathbf{N})$.

If $g_{i}(u)>0$ and $g_{i}^{\prime}(u)<0(i=1,2)$, then we find for $u>0$,

$$
g_{1}(u) g_{2}(u)>0, \quad\left(g_{1}(u) g_{2}(u)\right)^{\prime}=g_{1}^{\prime}(u) g_{2}(u)+g_{1}(u) g_{2}^{\prime}(u)<0 .
$$

(iii) Therefore, for $\rho, \gamma, \sigma>0, \alpha>-\rho(\alpha \geq 0)$, we have $h(t)>0$ and $h^{\prime}(t)<0$ with $k(\sigma) \in$ $\mathbf{R}_{+}$, and then for $c>0$ and $n \in \mathbf{N}$, adding $\sigma \leq 1$, we have

$$
h(c V(y)) V^{\sigma-1}(y)>0, \quad \frac{d}{d y} h(c V(y)) V^{\sigma-1}(y)<0 \quad(y \in(n-1, n)) .
$$

Lemma 1 If $g(t)(>0)$ is decreasing in $\mathbf{R}_{+}$and strictly decreasing in $\left[n_{0}, \infty\right)\left(n_{0} \in \mathbf{N}\right)$ and satisfies $\int_{0}^{\infty} g(t) d t \in \mathbf{R}_{+}$, then we have

$$
\int_{1}^{\infty} g(t) d t<\sum_{n=1}^{\infty} g(n)<\int_{0}^{\infty} g(t) d t
$$

Proof Since we have

$$
\begin{aligned}
& \int_{n}^{n+1} g(t) d t \leq g(n) \leq \int_{n-1}^{n} g(t) d t \quad\left(n=1, \ldots, n_{0}\right), \\
& \int_{n_{0}+1}^{n_{0}+2} g(t) d t<g\left(n_{0}+1\right)<\int_{n_{0}}^{n_{0}+1} g(t) d t
\end{aligned}
$$

it follows that

$$
0<\int_{1}^{n_{0}+2} g(t) d t<\sum_{n=1}^{n_{0}+1} g(n)<\sum_{n=1}^{n_{0}+1} \int_{n-1}^{n} g(t) d t=\int_{0}^{n_{0}+1} g(t) d t<\infty .
$$

In the same way, we still have

$$
0<\int_{n_{0}+2}^{\infty} g(t) d t \leq \sum_{n=n_{0}+2}^{\infty} g(n) \leq \int_{n_{0}+1}^{\infty} g(t) d t<\infty
$$

Hence, adding these two inequalities, we have (13). 
Lemma 2 For $\gamma, \rho>0, \alpha>-\rho(\alpha \geq 0)$, and $0<\sigma \leq 1$, define the following weight coefficients:

$$
\begin{aligned}
& \omega_{\delta}(\sigma, x):=\sum_{n=1}^{\infty} \frac{\operatorname{sech}\left(\rho\left(U^{\delta}(x) V_{n}\right)^{\gamma}\right)}{e^{\alpha\left(U^{\delta}(x) V_{n}\right)^{\gamma}}} \frac{U^{\delta \sigma}(x) v_{n}}{V_{n}^{1-\sigma}}, \quad x \in \mathbf{R}_{+}, \\
& \varpi_{\delta}(\sigma, n):=\int_{0}^{\infty} \frac{\sec h\left(\rho\left(U^{\delta}(x) V_{n}\right)^{\gamma}\right)}{e^{\alpha\left(U^{\delta}(x) V_{n}\right)^{\gamma}}} \frac{V_{n}^{\sigma} \mu(x)}{U^{1-\delta \sigma}(x)} d x, \quad n \in \mathbf{N} .
\end{aligned}
$$

Then, we have the following inequalities:

$$
\begin{aligned}
& \omega_{\delta}(\sigma, x)<k(\sigma) \quad\left(x \in \mathbf{R}_{+}\right), \\
& \varpi_{\delta}(\sigma, n) \leq k(\sigma) \quad(n \in \mathbf{N}),
\end{aligned}
$$

where $k(\sigma)$ is defined in (12).

Proof Since $V_{n}=V(n)$ and $V^{\prime}(t)=v_{n}$ for $t \in(n-1, n)$, by Example 1(iii) and the proof of Lemma 1 we have

$$
\begin{aligned}
& \frac{\operatorname{sech}\left(\rho\left(U^{\delta}(x) V_{n}\right)^{\gamma}\right) v_{n}}{e^{\alpha\left(U^{\delta}(x) V_{n}\right)^{\gamma}} V_{n}^{1-\sigma}} \\
& =\frac{\sec h\left(\rho\left(U^{\delta}(x) V(n)\right)^{\gamma}\right) v_{n}}{e^{\alpha\left(U^{\delta}(x) V(n)\right)^{\gamma}} V^{1-\sigma}(n)} \\
& <\int_{n-1}^{n} \frac{\operatorname{sech}\left(\rho\left(U^{\delta}(x) V(t)\right)^{\gamma}\right)}{e^{\alpha\left(U^{\delta}(x) V(t)\right)^{\gamma}}} \frac{V^{\prime}(t)}{V^{1-\sigma}(t)} d t \quad(n \in \mathbf{N}), \\
& \omega_{\delta}(\sigma, x)<\sum_{n=1}^{\infty} \int_{n-1}^{n} \frac{\operatorname{sech}\left(\rho\left(U^{\delta}(x) V(t)\right)^{\gamma}\right)}{e^{\alpha\left(U^{\delta}(x) V(t)\right)^{\gamma}}} \frac{U^{\delta \sigma}(x) V^{\prime}(t)}{V^{1-\sigma}(t)} d t \\
& =\int_{0}^{\infty} \frac{\operatorname{sech}\left(\rho\left(U^{\delta}(x) V(t)\right)^{\gamma}\right)}{e^{\alpha\left(U^{\delta}(x) V(t)\right)^{\gamma}}} \frac{U^{\delta \sigma}(x) V^{\prime}(t)}{V^{1-\sigma}(t)} d t .
\end{aligned}
$$

Setting $u=U^{\delta}(x) V(t)$, by (12) we find

$$
\begin{aligned}
\omega_{\delta}(\sigma, x) & <\int_{0}^{U^{\delta}(x) V(\infty)} \frac{\operatorname{sech}\left(\rho u^{\gamma}\right)}{e^{\alpha u^{\gamma}}} \frac{U^{\delta \sigma}(x) U^{-\delta}(x)}{\left(u U^{-\delta}(x)\right)^{1-\sigma}} d u \\
& \leq \int_{0}^{\infty} \frac{\operatorname{sech}\left(\rho u^{\gamma}\right)}{e^{\alpha u^{\gamma}}} u^{\sigma-1} d u \\
& =k(\sigma) .
\end{aligned}
$$

Hence, (16) follows.

Setting $u=V_{n} U^{\delta}(x)$ in (15), we find $d u=\delta V_{n} U^{\delta-1}(x) \mu(x) d x$ and

$$
\begin{aligned}
\varpi_{\delta}(\sigma, n) & =\frac{1}{\delta} \int_{V_{n} U^{\delta}(0)}^{V_{n} U^{\delta}(\infty)} \frac{\operatorname{sech}\left(\rho u^{\gamma}\right)}{e^{\alpha u^{\gamma}}} \frac{V_{n}^{\sigma} V_{n}^{-1}\left(V_{n}^{-1} u\right)^{\frac{1}{\delta}-1}}{\left(V_{n}^{-1} u\right)^{\frac{1}{\delta}-\sigma}} d u \\
& =\frac{1}{\delta} \int_{V_{n} U^{\delta}(0)}^{V_{n} u^{\delta}(\infty)} \frac{\operatorname{sech}\left(\rho u^{\gamma}\right)}{e^{\alpha u^{\gamma}}} u^{\sigma-1} d u .
\end{aligned}
$$


If $\delta=1$, then

$$
\varpi_{1}(\sigma, n)=\int_{0}^{V_{n} U(\infty)} \frac{\sec h\left(\rho u^{\gamma}\right)}{e^{\alpha u^{\gamma}}} u^{\sigma-1} d u \leq \int_{0}^{\infty} \frac{\operatorname{sech}\left(\rho u^{\gamma}\right)}{e^{\alpha u^{\gamma}}} u^{\sigma-1} d u
$$

if $\delta=-1$, then

$$
\varpi_{-1}(\sigma, n)=-\int_{\infty}^{V_{n} U^{-1}(\infty)} \frac{\sec h\left(\rho u^{\gamma}\right)}{e^{\alpha u^{\gamma}}} u^{\sigma-1} d u \leq \int_{0}^{\infty} \frac{\sec h\left(\rho u^{\gamma}\right)}{e^{\alpha u^{\gamma}}} u^{\sigma-1} d u
$$

Then by (12) we have (17).

Remark 1 We do not need $\sigma \leq 1$ to obtain (17). If $U(\infty)=\infty$, then we have

$$
\varpi_{\delta}(\sigma, n)=k(\sigma) \quad(n \in \mathbf{N}) .
$$

For example, set $\mu(t)=\frac{1}{(1+t)^{\beta}}(t>0 ; 0 \leq \beta \leq 1)$. Then, for $x \geq 0$, we find

$$
U(x)=\int_{0}^{x} \frac{d t}{(1+t)^{\beta}}= \begin{cases}\frac{(1+x)^{1-\beta}-1}{1-\beta}, & 0 \leq \beta<1,<\infty \\ \ln (1+x), & \beta=1\end{cases}
$$

and $U(\infty)=\int_{0}^{\infty} \frac{d t}{(1+t)^{\beta}}=\infty$

Lemma 3 If $\gamma, \rho>0, \alpha>-\rho(\alpha \geq 0), 0<\sigma \leq 1$, then there exists $n_{0} \in \mathbf{N}$ such that $v_{n} \geq v_{n+1}$ $\left(n \in\left\{n_{0}, n_{0}+1, \ldots\right\}\right)$, and $V(\infty)=\infty$. Moreover, then

(i) for $x \in \mathbf{R}_{+}$, we have

$$
k(\sigma)\left(1-\theta_{\delta}(\sigma, x)\right)<\omega_{\delta}(\sigma, x)
$$

where $\theta_{\delta}(\sigma, x)=O\left((U(x))^{\delta \sigma}\right) \in(0,1)$;

(ii) for any $b>0$, we have

$$
\sum_{n=1}^{\infty} \frac{v_{n}}{V_{n}^{1+b}}=\frac{1}{b}\left(\frac{1}{V_{n_{0}}^{b}}+b O(1)\right)
$$

Proof By Example 1(iii) we have

$$
\begin{aligned}
\omega_{\delta}(\sigma, x) & =\sum_{n=1}^{\infty} \frac{\operatorname{sech}\left(\rho\left(U^{\delta}(x) V_{n}\right)^{\gamma}\right)}{e^{\alpha\left(U^{\delta}(x) V_{n}\right)^{\gamma}}} \frac{U^{\delta \sigma}(x) v_{n}}{V_{n}^{1-\sigma}} \\
& \geq \sum_{n=n_{0}}^{\infty} \int_{n}^{n+1} \frac{\operatorname{sech}\left(\rho\left(U^{\delta}(x) V(n)\right)^{\gamma}\right)}{e^{\alpha\left(U^{\delta}(x) V(n)\right)^{\gamma}}} \frac{U^{\delta \sigma}(x) v_{n+1} d t}{(V(n))^{1-\sigma}} \\
& >\sum_{n=n_{0}}^{\infty} \int_{n}^{n+1} \frac{\operatorname{sech}\left(\rho\left(U^{\delta}(x) V(t)\right)^{\gamma}\right)}{e^{\alpha\left(U^{\delta}(x) V(t)\right)^{\gamma}}} \frac{U^{\delta \sigma}(x) V^{\prime}(t)}{(V(t))^{1-\sigma}} d t \\
& =\int_{n_{0}}^{\infty} \frac{\operatorname{sech}\left(\rho\left(U^{\delta}(x) V(t)\right)^{\gamma}\right)}{e^{\alpha\left(U^{\delta}(x) V(t)\right)^{\gamma}}} \frac{U^{\delta \sigma}(x) V^{\prime}(t)}{(V(t))^{1-\sigma}} d t .
\end{aligned}
$$


Setting $u=U^{\delta}(x) V(t)$, in view of $V(\infty)=\infty$, by (12) we find

$$
\begin{aligned}
& \omega_{\delta}(\sigma, x)>\int_{U^{\delta}(x) V_{n_{0}}}^{\infty} \frac{\sec h\left(\rho u^{\gamma}\right)}{e^{\alpha u^{\gamma}}} u^{\sigma-1} d u \\
& =k(\sigma)-\int_{0}^{U^{\delta}(x) V_{n_{0}}} \frac{\sec h\left(\rho u^{\gamma}\right)}{e^{\alpha u^{\gamma}}} u^{\sigma-1} d u=k(\sigma)\left(1-\theta_{\delta}(\sigma, x)\right), \\
& \theta_{\delta}(\sigma, x):=\frac{1}{k(\sigma)} \int_{0}^{U^{\delta}(x) V_{n_{0}}} \frac{\sec h\left(\rho u^{\gamma}\right)}{e^{\alpha u^{\gamma}}} u^{\sigma-1} d u \in(0,1) .
\end{aligned}
$$

Since $F(u)=\frac{\sec h\left(\rho u^{\gamma}\right)}{e^{\alpha u^{\gamma}}}$ is continuous in $(0, \infty)$ and satisfies $F(u) \rightarrow 1\left(u \rightarrow 0^{+}\right), F(u) \rightarrow$ $0(u \rightarrow \infty)$, there exists a constant $L>0$ such that $F(u) \leq L$, namely, $\frac{\sec h\left(\rho u^{\gamma}\right)}{e^{\alpha u^{\gamma}}} \leq L(u \in$ $(0, \infty))$. Hence, we find

$$
0<\theta_{\delta}(\sigma, x) \leq \frac{L}{k(\sigma)} \int_{0}^{U^{\delta}(x) V_{n_{0}}} u^{\sigma-1} d u=\frac{L\left(U^{\delta}(x) V_{n_{0}}\right)^{\sigma}}{k(\sigma) \sigma}
$$

and then (19) follows.

For $b>0$, we find

$$
\begin{aligned}
\sum_{n=1}^{\infty} \frac{v_{n}}{V_{n}^{1+b}} & =\sum_{n=1}^{n_{0}} \frac{v_{n}}{V_{n}^{1+b}}+\sum_{n=n_{0}+1}^{\infty} \frac{v_{n}}{V^{1+b}(n)} \\
& <\sum_{n=1}^{n_{0}} \frac{v_{n}}{V_{n}^{1+b}}+\sum_{n=n_{0}+1}^{\infty} \int_{n-1}^{n} \frac{V^{\prime}(x)}{V^{1+b}(x)} d x \\
& =\sum_{n=1}^{n_{0}} \frac{v_{n}}{V_{n}^{1+b}}+\int_{n_{0}}^{\infty} \frac{d V(x)}{V^{1+b}(x)}=\sum_{n=1}^{n_{0}} \frac{v_{n}}{V_{n}^{1+b}}+\frac{1}{b V^{b}\left(n_{0}\right)} \\
& =\frac{1}{b}\left(\frac{1}{V_{n_{0}}^{b}}+b \sum_{n=1}^{n_{0}} \frac{v_{n}}{\left.V_{n}^{1+b}\right)},\right. \\
\sum_{n=1}^{\infty} \frac{v_{n}}{V_{n}^{1+b}} & \geq \sum_{n=n_{0}}^{\infty} \int_{n}^{n+1} \frac{v_{n+1}}{V^{1+b}(n)} d x>\sum_{n=n_{0}}^{\infty} \int_{n}^{n+1} \frac{V^{\prime}(x) d x}{V^{1+b}(x)} \\
& =\int_{n_{0}}^{\infty} \frac{d V(x)}{V^{1+b}(x)}=\frac{1}{b V^{b}\left(n_{0}\right)}=\frac{1}{b V_{n_{0}}^{b}} .
\end{aligned}
$$

Hence, we have (20).

Note For example, $v_{n}=\frac{1}{n^{\beta}}(n \in \mathbf{N} ; 0 \leq \beta \leq 1)$ satisfies the conditions of Lemma 3 (for $\left.n_{0}=1\right)$.

\section{Main results and operator expressions}

Theorem 1 If $\gamma, \rho>0, \alpha>-\rho(\alpha \geq 0), 0<\sigma \leq 1, k(\sigma)$ is defined in by (12), then for $p>1$, $0<\|f\|_{p, \Phi_{\delta}},\|a\|_{q, \Psi}<\infty$, we have the following equivalent inequalities:

$$
I:=\sum_{n=1}^{\infty} \int_{0}^{\infty} \frac{\sec h\left(\rho\left(U^{\delta}(x) V_{n}\right)^{\gamma}\right)}{e^{\alpha\left(U^{\delta}(x) V_{n}\right)^{\gamma}}} a_{n} f(x) d x<k(\sigma)\|f\|_{p, \Phi_{\delta}}\|a\|_{q, \Psi}
$$




$$
\begin{aligned}
& J_{1}:=\sum_{n=1}^{\infty} \frac{v_{n}}{V_{n}^{1-p \sigma}}\left[\int_{0}^{\infty} \frac{\operatorname{sech}\left(\rho\left(U^{\delta}(x) V_{n}\right)^{\gamma}\right)}{e^{\alpha\left(U^{\delta}(x) V_{n}\right)^{\gamma}}} f(x) d x\right]^{p} \\
& <k(\sigma)\|f\|_{p, \Phi_{\delta}}, \\
& J_{2}:=\left\{\int_{0}^{\infty} \frac{\mu(x)}{U^{1-q \delta \sigma}(x)}\left[\sum_{n=1}^{\infty} \frac{\operatorname{sech}\left(\rho\left(U^{\delta}(x) V_{n}\right)^{\gamma}\right)}{e^{\alpha\left(U^{\delta}(x) V_{n}\right)^{\gamma}}} a_{n}\right]^{q} d x\right\}^{\frac{1}{q}} \\
& <k(\sigma)\|a\|_{q, \Psi} .
\end{aligned}
$$

Proof By Hölder's inequality with weight (see [41]), we have

$$
\begin{aligned}
{\left[\int_{0}^{\infty} \frac{\sec h\left(\rho\left(U^{\delta}(x) V_{n}\right)^{\gamma}\right)}{\left.e^{\alpha\left(U^{\delta}(x) V_{n}\right)^{\gamma}} f(x) d x\right]^{p}}\right.} \\
=\left[\int_{0}^{\infty} \frac{\operatorname{sech}\left(\rho\left(U^{\delta}(x) V_{n}\right)^{\gamma}\right)}{e^{\alpha\left(U^{\delta}(x) V_{n}\right)^{\gamma}}}\left(\frac{U^{\frac{1-\delta \sigma}{q}}(x) f(x)}{V_{n}^{\frac{1-\sigma}{p}} \mu^{\frac{1}{q}}(x)}\right)\left(\frac{V_{n}^{\frac{1-\sigma}{p}} \mu^{\frac{1}{q}}(x)}{U^{\frac{1-\delta \sigma}{q}}(x)}\right) d x\right]^{p} \\
\leq \int_{0}^{\infty} \frac{\operatorname{sech}\left(\rho\left(U^{\delta}(x) V_{n}\right)^{\gamma}\right)}{e^{\alpha\left(U^{\delta}(x) V_{n}\right)^{\gamma}}}\left(\frac{U^{\frac{p(1-\delta \sigma)}{q}}(x) f^{p}(x)}{V_{n}^{1-\sigma} \mu^{\frac{p}{q}}(x)}\right) d x \\
\quad \times\left[\int_{0}^{\infty} \frac{\sec h\left(\rho\left(U^{\delta}(x) V_{n}\right)^{\gamma}\right)}{e^{\alpha\left(U^{\delta}(x) V_{n}\right)^{\gamma}}} \frac{V_{n}^{(1-\sigma)(p-1)} \mu(x)}{U^{1-\delta \sigma}(x)} d x\right]^{p-1} \\
=\frac{\left(\varpi_{\delta}(\sigma, n)\right)^{p-1}}{V_{n}^{p \sigma-1} v_{n}} \int_{0}^{\infty} \frac{\operatorname{sech}\left(\rho\left(U^{\delta}(x) V_{n}\right)^{\gamma}\right)}{e^{\alpha\left(U^{\delta}(x) V_{n}\right)^{\gamma}}} \frac{U^{(1-\delta \sigma)(p-1)}(x) v_{n}}{V_{n}^{1-\sigma} \mu^{p-1}(x)} f^{p}(x) d x .
\end{aligned}
$$

In view of (17) and the Lebesgue term-by-term integration theorem (see [42]), we find

$$
\begin{aligned}
J_{1} & \leq(k(\sigma))^{\frac{1}{q}}\left[\sum_{n=1}^{\infty} \int_{0}^{\infty} \frac{\operatorname{sech}\left(\rho\left(U^{\delta}(x) V_{n}\right)^{\gamma}\right)}{e^{\alpha\left(U^{\delta}(x) V_{n}\right)^{\gamma}}} \frac{U^{(1-\delta \sigma)(p-1)}(x) v_{n}}{V_{n}^{1-\sigma} \mu^{p-1}(x)} f^{p}(x) d x\right]^{\frac{1}{p}} \\
& =(k(\sigma))^{\frac{1}{q}}\left[\int_{0}^{\infty} \sum_{n=1}^{\infty} \frac{\sec h\left(\rho\left(U^{\delta}(x) V_{n}\right)^{\gamma}\right)}{e^{\alpha\left(U^{\delta}(x) V_{n}\right)^{\gamma}}} \frac{U^{(1-\delta \sigma)(p-1)}(x) v_{n}}{V_{n}^{1-\sigma} \mu^{p-1}(x)} f^{p}(x) d x\right]^{\frac{1}{p}} \\
& =(k(\sigma))^{\frac{1}{q}}\left[\int_{0}^{\infty} \omega_{\delta}(\sigma, x) \frac{U^{p(1-\delta \sigma)-1}(x)}{\mu^{p-1}(x)} f^{p}(x) d x\right]^{\frac{1}{p}} .
\end{aligned}
$$

Then by (16) we have (22).

By Hölder's inequality (see [41]) we have

$$
\begin{aligned}
I & =\sum_{n=1}^{\infty}\left[\frac{v_{n}^{\frac{1}{p}}}{V_{n}^{\frac{1}{p}-\sigma}} \int_{0}^{\infty} \frac{\operatorname{sech}\left(\rho\left(U^{\delta}(x) V_{n}\right)^{\gamma}\right)}{e^{\alpha\left(U^{\delta}(x) V_{n}\right)^{\gamma}}} f(x) d x\right]\left(\frac{V_{n}^{\frac{1}{p}-\sigma} a_{n}}{v_{n}^{\frac{1}{p}}}\right) \\
& \leq J_{1}\|a\|_{q, \Psi} .
\end{aligned}
$$

Then by (22) we have (21). On the other hand, assuming that (21) is valid, we set

$$
a_{n}:=\frac{v_{n}}{V_{n}^{1-p \sigma}}\left[\int_{0}^{\infty} \frac{\operatorname{sech}\left(\rho\left(U^{\delta}(x) V_{n}\right)^{\gamma}\right)}{e^{\alpha\left(U^{\delta}(x) V_{n}\right)^{\gamma}}} f(x) d x\right]^{p-1}, \quad n \in \mathbf{N}
$$


Then we find $J_{1}^{p}=\|a\|_{q, \Psi}^{q}$. If $J_{1}=0$, then (22) is trivially valid; if $J_{1}=\infty$, then (22) keeps impossible. Suppose that $0<J_{1}<\infty$. By (21) we have

$$
\|a\|_{q, \Psi}^{q}=J_{1}^{p}=I<k(\sigma)\|f\|_{p, \Phi_{\delta}}\|a\|_{q, \Psi}, \quad\|a\|_{q, \Psi}^{q-1}=J_{1}<k(\sigma)\|f\|_{p, \Phi_{\delta}},
$$

and then (22) follows, which is equivalent to (21).

Again by Hölder's inequality with weight we have

$$
\begin{aligned}
& {\left[\sum_{n=1}^{\infty} \frac{\sec h\left(\rho\left(U^{\delta}(x) V_{n}\right)^{\gamma}\right)}{e^{\alpha\left(U^{\delta}(x) V_{n}\right)^{\gamma}} a_{n}}\right]^{q} } \\
&= {\left[\sum_{n=1}^{\infty} \frac{\operatorname{sech}\left(\rho\left(U^{\delta}(x) V_{n}\right)^{\gamma}\right)}{e^{\alpha\left(U^{\delta}(x) V_{n}\right)^{\gamma}}}\left(\frac{U^{\frac{1-\delta \sigma}{q}}(x) v_{n}^{\frac{1}{p}}}{V_{n}^{\frac{1-\sigma}{p}}}\right)\left(\frac{V_{n}^{\frac{1-\sigma}{p}} a_{n}}{U^{\frac{1-\delta \sigma}{q}}(x) v_{n}^{\frac{1}{p}}}\right)\right]^{q} } \\
& \leq {\left[\sum_{n=1}^{\infty} \frac{\operatorname{sech}\left(\rho\left(U^{\delta}(x) V_{n}\right)^{\gamma}\right)}{e^{\alpha\left(U^{\delta}(x) V_{n}\right)^{\gamma}}} \frac{U^{(1-\delta \sigma)(p-1)}(x) v_{n}}{V_{n}^{1-\sigma}}\right]^{q-1} } \\
& \times \sum_{n=1}^{\infty} \frac{\operatorname{sech}\left(\rho\left(U^{\delta}(x) V_{n}\right)^{\gamma}\right)}{e^{\alpha\left(U^{\delta}(x) V_{n}\right)^{\gamma}}} \frac{V_{n}^{\frac{q(1-\sigma)}{p}}}{U^{1-\delta \sigma}(x) v_{n}^{q-1}} a_{n}^{q} \\
&= \frac{\left(\omega_{\delta}(\sigma, x)\right)^{q-1}}{U^{q \delta \sigma-1}(x) \mu(x)} \sum_{n=1}^{\infty} \frac{\operatorname{sech}\left(\rho\left(U^{\delta}(x) V_{n}\right)^{\gamma}\right)}{e^{\alpha\left(U^{\delta}(x) V_{n}\right)^{\gamma}} \frac{V_{n}^{(1-\sigma)(q-1)} \mu(x)}{U^{1-\delta \sigma}(x) v_{n}^{q-1}} a_{n}^{q} .}
\end{aligned}
$$

Then by (16) and the Lebesgue term-by-term integration theorem it follows that

$$
\begin{aligned}
J_{2} & <(k(\sigma))^{\frac{1}{p}}\left\{\int_{0}^{\infty} \sum_{n=1}^{\infty} \frac{\operatorname{sech}\left(\rho\left(U^{\delta}(x) V_{n}\right)^{\gamma}\right)}{e^{\alpha\left(U^{\delta}(x) V_{n}\right)^{\gamma}}} \frac{V_{n}^{(1-\sigma)(q-1)} \mu(x)}{U^{1-\delta \sigma}(x) v_{n}^{q-1}} a_{n}^{q} d x\right\}^{\frac{1}{q}} \\
& =(k(\sigma))^{\frac{1}{p}}\left\{\sum_{n=1}^{\infty} \int_{0}^{\infty} \frac{\sec h\left(\rho\left(U^{\delta}(x) V_{n}\right)^{\gamma}\right)}{e^{\alpha\left(U^{\delta}(x) V_{n}\right)^{\gamma}}} \frac{V_{n}^{(1-\sigma)(q-1)} \mu(x)}{U^{1-\delta \sigma}(x) v_{n}^{q-1}} a_{n}^{q} d x\right\}^{\frac{1}{q}} \\
& =(k(\sigma))^{\frac{1}{p}}\left\{\sum_{n=1}^{\infty} \varpi_{\delta}(\sigma, n) \frac{V_{n}^{q(1-\sigma)-1}}{v_{n}^{q-1}} a_{n}^{q}\right\}^{\frac{1}{q}} .
\end{aligned}
$$

Then by (17) we have (23).

By Hölder's inequality we have

$$
\begin{aligned}
I & =\int_{0}^{\infty}\left(\frac{U^{\frac{1}{q}-\delta \sigma}(x)}{\mu^{\frac{1}{q}}(x)} f(x)\right)\left[\frac{\mu^{\frac{1}{q}}(x)}{U^{\frac{1}{q}-\delta \sigma}(x)} \sum_{n=1}^{\infty} \frac{\operatorname{sech}\left(\rho\left(U^{\delta}(x) V_{n}\right)^{\gamma}\right)}{e^{\alpha\left(U^{\delta}(x) V_{n}\right)^{\gamma}}} a_{n}\right] d x \\
& \leq\|f\|_{p, \Phi_{\delta}} J_{2} .
\end{aligned}
$$

Then by (23) we have (21). On the other hand, assuming that (23) is valid, we set

$$
f(x):=\frac{\mu(x)}{U^{1-q \delta \sigma}(x)}\left[\sum_{n=1}^{\infty} \frac{\operatorname{sech}\left(\rho\left(U^{\delta}(x) V_{n}\right)^{\gamma}\right)}{e^{\alpha\left(U^{\delta}(x) V_{n}\right)^{\gamma}}} a_{n}\right]^{q-1}, \quad x \in \mathbf{R}_{+} .
$$


Then we find $J_{2}^{q}=\|f\|_{p, \Phi_{\delta}}^{p}$. If $J_{2}=0$, then (23) is trivially valid; if $J_{2}=\infty$, then (23) keeps impossible. Suppose that $0<J_{2}<\infty$. By (21) we have

$$
\|f\|_{p, \Phi_{\delta}}^{p}=J_{2}^{q}=I<k(\sigma)\|f\|_{p, \Phi_{\delta}}\|a\|_{q, \Psi}, \quad\|f\|_{p, \Phi_{\delta}}^{p-1}=J_{2}<k(\sigma)\|a\|_{q, \Psi},
$$

and then (23) follows, which is equivalent to (21).

Therefore, (21), (22), and (23) are equivalent.

Theorem 2 With the assumptions of Theorem 1 , if there exists $n_{0} \in \mathbf{N}$ such that $v_{n} \geq v_{n+1}$ $\left(n \in\left\{n_{0}, n_{0}+1, \ldots\right\}\right)$ and $U(\infty)=V(\infty)=\infty$, then the constant factor $k(\sigma)$ in (21), (22), and (23) is the best possible.

Proof For $\varepsilon \in(0, q \sigma)$, we set $\tilde{\sigma}=\sigma-\frac{\varepsilon}{q}$ and $\tilde{f}=\tilde{f}(x), x \in \mathbf{R}_{+}, \tilde{a}=\left\{\tilde{a}_{n}\right\}_{n=1}^{\infty}$,

$$
\begin{aligned}
& \tilde{f}(x)= \begin{cases}U^{\delta(\tilde{\sigma}+\varepsilon)-1}(x) \mu(x), & 0<x^{\delta} \leq 1, \\
0, & x^{\delta}>0,\end{cases} \\
& \tilde{a}_{n}=V_{n}^{\tilde{\sigma}-1} v_{n}=V_{n}^{\sigma-\frac{\varepsilon}{q}-1} v_{n}, \quad n \in \mathbf{N} .
\end{aligned}
$$

Then for $\delta= \pm 1$, since $U(\infty)=\infty$, we find

$$
\int_{\left\{x>0 ; 0<x^{\delta} \leq 1\right\}} \frac{\mu(x)}{U^{1-\delta \varepsilon}(x)} d x=\frac{1}{\varepsilon} U^{\delta \varepsilon}(1) .
$$

By (20), (32), and (19) we obtain

$$
\begin{aligned}
& \|\tilde{f}\|_{p, \Phi_{\delta}}\|\tilde{a}\|_{q, \Psi}=\left(\int_{\left\{x>0 ; 0<x^{\delta} \leq 1\right\}} \frac{\mu(x) d x}{U^{1-\delta \varepsilon}(x)}\right)^{\frac{1}{p}}\left(\sum_{n=1}^{\infty} \frac{v_{n}}{V_{n}^{1+\varepsilon}}\right)^{\frac{1}{q}} \\
& =\frac{1}{\varepsilon} U^{\frac{\delta \varepsilon}{p}}(1)\left(\frac{1}{V_{n_{0}}^{\varepsilon}}+\varepsilon O(1)\right)^{\frac{1}{q}}, \\
& \tilde{I}:=\int_{0}^{\infty} \sum_{n=1}^{\infty} \frac{\operatorname{sech}\left(\rho\left(U^{\delta}(x) V_{n}\right)^{\gamma}\right)}{e^{\alpha\left(U^{\delta}(x) V_{n}\right)^{\gamma}}} \tilde{a}_{n} \tilde{f}(x) d x \\
& =\int_{\left\{x>0 ; 0<x^{\delta} \leq 1\right\}} \sum_{n=1}^{\infty} \frac{\operatorname{sech}\left(\rho\left(U^{\delta}(x) V_{n}\right)^{\gamma}\right)}{e^{\alpha\left(U^{\delta}(x) V_{n}\right)^{\gamma}}} \frac{V_{n}^{\tilde{\sigma}-1} v_{n} \mu(x)}{U^{1-\delta(\tilde{\sigma}+\varepsilon)}(x)} d x \\
& =\int_{\left\{x>0 ; 0<x^{\delta} \leq 1\right\}} \omega_{\delta}(\tilde{\sigma}, x) \frac{\mu(x)}{U^{1-\delta \varepsilon}(x)} d x \\
& \geq k(\tilde{\sigma}) \int_{\left\{x>0 ; 0<x^{\delta} \leq 1\right\}}\left(1-\theta_{\delta}(\tilde{\sigma}, x)\right) \frac{\mu(x)}{U^{1-\delta \varepsilon}(x)} d x \\
& =k(\tilde{\sigma}) \int_{\left\{x>0 ; 0<x^{\delta} \leq 1\right\}}\left(1-O\left((U(x))^{\delta\left(\sigma-\frac{\varepsilon}{q}\right)}\right)\right) \frac{\mu(x)}{U^{1-\delta \varepsilon}(x)} d x \\
& =k(\tilde{\sigma})\left[\int_{\left\{x>0 ; 0<x^{\delta} \leq 1\right\}} \frac{\mu(x)}{U^{1-\delta \varepsilon}(x)} d x-\int_{\left\{x>0 ; 0<x^{\delta} \leq 1\right\}} O\left(\frac{\mu(x)}{U^{1-\delta\left(\sigma+\frac{\varepsilon}{p}\right)}(x)}\right) d x\right] \\
& =\frac{1}{\varepsilon} k\left(\sigma-\frac{\varepsilon}{q}\right)\left(U^{\delta \varepsilon}(1)-\varepsilon O(1)\right) \text {. }
\end{aligned}
$$


If there exists a positive constant $K \leq k(\sigma)$ such that (21) is valid when replacing $k(\sigma)$ by $K$, then, in particular, by the Lebesgue term-by-term integration theorem we have $\varepsilon \tilde{I}<$ $\varepsilon K\|\tilde{f}\|_{p, \Phi_{\delta}}\|\tilde{a}\|_{q, \Psi}$, namely,

$$
k\left(\sigma-\frac{\varepsilon}{q}\right)\left(U^{\delta \varepsilon}(1)-\varepsilon O(1)\right)<K \cdot U^{\frac{\delta \varepsilon}{p}}(1)\left(\frac{1}{V_{n_{0}}^{\varepsilon}}+\varepsilon O(1)\right)^{\frac{1}{q}} .
$$

It follows that $k(\sigma) \leq K\left(\varepsilon \rightarrow 0^{+}\right)$. Hence, $K=k(\sigma)$ is the best possible constant factor of (21).

The constant factor $k(\sigma)$ in (22) ((23)) is still the best possible. Otherwise, we would reach a contradiction by (26) ((29)) that the constant factor in (21) is not the best possible.

For $p>1$, we find $\Psi^{1-p}(n)=\frac{v_{n}}{V_{n}^{1-p \sigma}}(n \in \mathbf{N}), \Phi_{\delta}^{1-q}(x)=\frac{\mu(x)}{U^{1-q \delta \sigma}(x)}\left(x \in \mathbf{R}_{+}\right)$and define the following real normed spaces:

$$
\begin{aligned}
& L_{p, \Phi_{\delta}}\left(\mathbf{R}_{+}\right)=\left\{f ; f=f(x), x \in \mathbf{R}_{+},\|f\|_{p, \Phi_{\delta}}<\infty\right\}, \\
& l_{q, \Psi}=\left\{a ; a=\left\{a_{n}\right\}_{n=1}^{\infty},\|a\|_{q, \Psi}<\infty\right\}, \\
& L_{q, \Phi_{\delta}^{1-q}}\left(\mathbf{R}_{+}\right)=\left\{h ; h=h(x), x \in \mathbf{R}_{+},\|h\|_{q, \Phi_{\delta}^{1-q}}<\infty\right\}, \\
& l_{p, \Psi 1-p}=\left\{c ; c=\left\{c_{n}\right\}_{n=1}^{\infty},\|c\|_{p, \Psi 1-p}<\infty\right\} .
\end{aligned}
$$

Assuming that $f \in L_{p, \Phi_{\delta}}\left(\mathbf{R}_{+}\right)$and setting

$$
c=\left\{c_{n}\right\}_{n=1}^{\infty}, \quad c_{n}:=\int_{0}^{\infty} \frac{\operatorname{sech}\left(\rho\left(U^{\delta}(x) V_{n}\right)^{\gamma}\right)}{e^{\alpha\left(U^{\delta}(x) V_{n}\right)^{\gamma}}} f(x) d x, \quad n \in \mathbf{N},
$$

we can rewrite (22) as $\|c\|_{p, \Psi^{1-p}}<k(\sigma)\|f\|_{p, \Phi_{\delta}}<\infty$, namely, $c \in l_{p, \Psi^{1-p}}$.

Definition 1 Define a half-discrete Hardy-Hilbert-type operator $T_{1}: L_{p, \Phi_{\delta}}\left(\mathbf{R}_{+}\right) \rightarrow l_{p, \Psi^{1-p}}$ as follows: For any $f \in L_{p, \Phi_{\delta}}\left(\mathbf{R}_{+}\right)$, the exists a unique representation $T_{1} f=c \in l_{p, \Psi^{1-p}}$. We define the formal inner product of $T_{1} f$ and $a=\left\{a_{n}\right\}_{n=1}^{\infty} \in l_{q, \Psi}$ as follows:

$$
\left(T_{1} f, a\right):=\sum_{n=1}^{\infty}\left[\int_{0}^{\infty} \frac{\operatorname{sech}\left(\rho\left(U^{\delta}(x) V_{n}\right)^{\gamma}\right)}{e^{\alpha\left(U^{\delta}(x) V_{n}\right)^{\gamma}}} f(x) d x\right] a_{n} .
$$

Then we can rewrite (21) and (22) as follows:

$$
\begin{aligned}
& \left(T_{1} f, a\right)<k(\sigma)\|f\|_{p, \Phi_{\delta}}\|a\|_{q, \Psi}, \\
& \left\|T_{1} f\right\|_{p, \Psi 1-p}<k(\sigma)\|f\|_{p, \Phi_{\delta}} .
\end{aligned}
$$

Define the norm of operator $T_{1}$ as follows:

$$
\left\|T_{1}\right\|:=\sup _{f(\not \theta) \in L_{p, \Phi_{\delta}}\left(\mathbf{R}_{+}\right)} \frac{\left\|T_{1} f\right\|_{p, \Psi^{1-p}}}{\|f\|_{p, \Phi_{\delta}}} .
$$


Then by (36) it follows that $\left\|T_{1}\right\| \leq k(\sigma)$. Since by Theorem 2 the constant factor in (36) is the best possible, we have

$$
\left\|T_{1}\right\|=k(\sigma)=\frac{2 \Gamma\left(\frac{\sigma}{\gamma}\right)}{\gamma(2 \rho)^{\sigma / \gamma}} \xi\left(\frac{\sigma}{\gamma}, \frac{\alpha+\rho}{2 \rho}\right) .
$$

Assuming that $a=\left\{a_{n}\right\}_{n=1}^{\infty} \in l_{q, \Psi}$ and setting

$$
h(x):=\sum_{n=1}^{\infty} \frac{\operatorname{sech}\left(\rho\left(U^{\delta}(x) V_{n}\right)^{\gamma}\right)}{e^{\alpha\left(U^{\delta}(x) V_{n}\right)^{\gamma}}} a_{n}, \quad x \in \mathbf{R}_{+},
$$

we can rewrite (23) as $\|h\|_{q, \Phi_{\delta}^{1-q}}<k(\sigma)\|a\|_{q, \Psi}<\infty$, namely, $h \in L_{q, \Phi_{\delta}^{1-q}}\left(\mathbf{R}_{+}\right)$.

Definition 2 Define a half-discrete Hardy-Hilbert-type operator $T_{2}: l_{q, \Psi} \rightarrow L_{q, \Phi_{\delta}^{1-q}}\left(\mathbf{R}_{+}\right)$ as follows: For any $a=\left\{a_{n}\right\}_{n=1}^{\infty} \in l_{q, \Psi}$, there exists a unique representation $T_{2} a=h \in$ $L_{q, \Phi_{\delta}^{1-q}}\left(\mathbf{R}_{+}\right)$. We define the formal inner product of $T_{2} a$ and $f \in L_{p, \Phi_{\delta}}\left(\mathbf{R}_{+}\right)$as follows:

$$
\left(T_{2} a, f\right):=\int_{0}^{\infty}\left[\sum_{n=1}^{\infty} \frac{\operatorname{sech}\left(\rho\left(U^{\delta}(x) V_{n}\right)^{\gamma}\right)}{e^{\alpha\left(U^{\delta}(x) V_{n}\right)^{\gamma}}} a_{n}\right] f(x) d x .
$$

Then we can rewrite (21) and (23) as follows:

$$
\begin{aligned}
& \left(T_{2} a, f\right)<k(\sigma)\|f\|_{p, \Phi_{\delta}}\|a\|_{q, \Psi}, \\
& \left\|T_{2} a\right\|_{q, \Phi_{\delta}^{1-q}}<k(\sigma)\|a\|_{q, \Psi} .
\end{aligned}
$$

Define the norm of operator $T_{2}$ as follows:

$$
\left\|T_{2}\right\|:=\sup _{a(\neq \theta) \in l_{q, \Psi}} \frac{\left\|T_{2} a\right\|_{q, \Phi_{\delta}^{1-q}}}{\|a\|_{q, \Psi}} .
$$

Then by (40) we find $\left\|T_{2}\right\| \leq k(\sigma)$. Since by Theorem 2 the constant factor in (40) is the best possible, we have

$$
\left\|T_{2}\right\|=k(\sigma)=\frac{2 \Gamma\left(\frac{\sigma}{\gamma}\right)}{\gamma(2 \rho)^{\sigma / \gamma}} \xi\left(\frac{\sigma}{\gamma}, \frac{\alpha+\rho}{2 \rho}\right)=\left\|T_{1}\right\|
$$

\section{Some equivalent reverse inequalities}

In the following, we also set

$$
\widetilde{\Phi}_{\delta}(x):=\left(1-\theta_{\delta}(\sigma, x)\right) \frac{U^{p(1-\delta \sigma)-1}(x)}{\mu^{p-1}(x)} \quad\left(x \in \mathbf{R}_{+}\right) .
$$

For $0<p<1$ or $p<0$, we still use the formal symbols $\|f\|_{p, \Phi_{\delta}},\|f\|_{p, \widetilde{\Phi}_{\delta}}$, and $\|a\|_{q, \Psi}$.

Theorem 3 If $\gamma, \rho>0, \alpha>-\rho(\alpha \geq 0), 0<\sigma \leq 1, k(\sigma)$ is defined in (12), there exists $n_{0} \in$ $\mathbf{N}$ such that $v_{n} \geq v_{n+1}\left(n \in\left\{n_{0}, n_{0}+1, \ldots\right\}\right)$, and $U(\infty)=V(\infty)=\infty$, then for $p<0,0<$ 
$\|f\|_{p, \Phi_{\delta}},\|a\|_{q, \Psi}<\infty$, we have the following equivalent inequalities with the best possible constant factor $k(\sigma)$ :

$$
\begin{aligned}
& I=\sum_{n=1}^{\infty} \int_{0}^{\infty} \frac{\sec h\left(\rho\left(U^{\delta}(x) V_{n}\right)^{\gamma}\right)}{e^{\alpha\left(U^{\delta}(x) V_{n}\right)^{\gamma}}} a_{n} f(x) d x>k(\sigma)\|f\|_{p, \Phi_{\delta}}\|a\|_{q, \Psi}, \\
& J_{1}=\sum_{n=1}^{\infty} \frac{v_{n}}{V_{n}^{1-p \sigma}}\left[\int_{0}^{\infty} \frac{\operatorname{sech}\left(\rho\left(U^{\delta}(x) V_{n}\right)^{\gamma}\right)}{e^{\alpha\left(U^{\delta}(x) V_{n}\right)^{\gamma}}} f(x) d x\right]^{p}>k(\sigma)\|f\|_{p, \Phi_{\delta}}, \\
& J_{2}=\left\{\int_{0}^{\infty} \frac{\mu(x)}{U^{1-q \delta \sigma}(x)}\left[\sum_{n=1}^{\infty} \frac{\operatorname{sech}\left(\rho\left(U^{\delta}(x) V_{n}\right)^{\gamma}\right)}{e^{\alpha\left(U^{\delta}(x) V_{n}\right)^{\gamma}}} a_{n}\right]^{q} d x\right\}^{\frac{1}{q}} \\
& >k(\sigma)\|a\|_{q, \Psi} \text {. }
\end{aligned}
$$

Proof By the reverse Hölder inequality with weight (see [41]), since $p<0$, similarly as obtaining (24) and (25), we have

$$
\begin{aligned}
& {\left[\int_{0}^{\infty} \frac{\sec h\left(\rho\left(U^{\delta}(x) V_{n}\right)^{\gamma}\right)}{\left.e^{\alpha\left(U^{\delta}(x) V_{n}\right)^{\gamma}} f(x) d x\right]^{p}}\right.} \\
& \quad \leq \frac{\left(\varpi_{\delta}(\sigma, n)\right)^{p-1}}{V_{n}^{p \sigma-1} v_{n}} \int_{0}^{\infty} \frac{\operatorname{sech}\left(\rho\left(U^{\delta}(x) V_{n}\right)^{\gamma}\right)}{e^{\alpha\left(U^{\delta}(x) V_{n}\right)^{\gamma}}} \frac{U^{(1-\delta \sigma)(p-1)}(x) v_{n}}{V_{n}^{1-\sigma} \mu^{p-1}(x)} f^{p}(x) d x .
\end{aligned}
$$

Then by (18) and the Lebesgue term-by-term integration theorem it follows that

$$
\begin{aligned}
J_{1} & \geq(k(\sigma))^{\frac{1}{q}}\left[\sum_{n=1}^{\infty} \int_{0}^{\infty} \frac{\operatorname{sech}\left(\rho\left(U^{\delta}(x) V_{n}\right)^{\gamma}\right)}{e^{\alpha\left(U^{\delta}(x) V_{n}\right)^{\gamma}}} \frac{U^{(1-\delta \sigma)(p-1)}(x) v_{n}}{V_{n}^{1-\sigma} \mu^{p-1}(x)} f^{p}(x) d x\right]^{\frac{1}{p}} \\
& =(k(\sigma))^{\frac{1}{q}}\left[\int_{0}^{\infty} \omega_{\delta}(\sigma, x) \frac{U^{p(1-\delta \sigma)-1}(x)}{\mu^{p-1}(x)} f^{p}(x) d x\right]^{\frac{1}{p}} .
\end{aligned}
$$

Then by (16) we have (43).

By the reverse Hölder inequality we have

$$
\begin{aligned}
I & =\sum_{n=1}^{\infty}\left[\frac{v_{n}^{\frac{1}{p}}}{V_{n}^{\frac{1}{p}-\sigma}} \int_{0}^{\infty} \frac{\operatorname{sech}\left(\rho\left(U^{\delta}(x) V_{n}\right)^{\gamma}\right)}{e^{\alpha\left(U^{\delta}(x) V_{n}\right)^{\gamma}}} f(x) d x\right]\left(\frac{V_{n}^{\frac{1}{p}-\sigma} a_{n}}{v_{n}^{\frac{1}{p}}}\right) \\
& \geq J_{1}\|a\|_{q, \Psi} .
\end{aligned}
$$

Then by (43) we have (42). On the other hand, assuming that (42) is valid, we set $a_{n}$ as in Theorem 1 . Then we find $J_{1}^{p}=\|a\|_{q, \Psi}^{q}$. If $J_{1}=\infty$, then (43) is trivially valid; if $J_{1}=0$, then (43) keeps impossible. Suppose that $0<J_{1}<\infty$. By (42) it follows that

$$
\begin{aligned}
& \|a\|_{q, \Psi}^{q}=J_{1}^{p}=I>k(\sigma)\|f\|_{p, \Phi_{\delta}}\|a\|_{q, \Psi}, \\
& \|a\|_{q, \Psi}^{q-1}=J_{1}>k(\sigma)\|f\|_{p, \Phi_{\delta}},
\end{aligned}
$$

and then (43) follows, which is equivalent to (42). 
Still by the reverse Hölder inequality with weight, since $0<q<1$, similarly as obtaining (27) and (28), we have

$$
\begin{aligned}
& {\left[\sum_{n=1}^{\infty} \frac{\operatorname{sech}\left(\rho\left(U^{\delta}(x) V_{n}\right)^{\gamma}\right)}{e^{\alpha\left(U^{\delta}(x) V_{n}\right)^{\gamma}}} a_{n}\right]^{q}} \\
& \quad \geq \frac{\left(\omega_{\delta}(\sigma, x)\right)^{q-1}}{U^{q \delta \sigma-1}(x) \mu(x)} \sum_{n=1}^{\infty} \frac{\operatorname{sech}\left(\rho\left(U^{\delta}(x) V_{n}\right)^{\gamma}\right)}{e^{\alpha\left(U^{\delta}(x) V_{n}\right)^{\gamma}}} \frac{V_{n}^{(1-\sigma)(q-1)} \mu(x)}{U^{1-\delta \sigma}(x) v_{n}^{q-1}} a_{n}^{q} .
\end{aligned}
$$

Then by (16) and the Lebesgue term-by-term integration theorem it follows that

$$
\begin{aligned}
J_{2}>(k(\sigma))^{\frac{1}{p}}\left\{\int_{0}^{\infty} \sum_{n=1}^{\infty} \frac{\operatorname{sech}\left(\rho\left(U^{\delta}(x) V_{n}\right)^{\gamma}\right)}{e^{\alpha\left(U^{\delta}(x) V_{n}\right)^{\gamma}}} \frac{V_{n}^{(1-\sigma)(q-1)} \mu(x)}{U^{1-\delta \sigma}(x) v_{n}^{q-1}} a_{n}^{q} d x\right\}^{\frac{1}{q}} \\
=(k(\sigma))^{\frac{1}{p}}\left\{\sum_{n=1}^{\infty} \varpi_{\delta}(\sigma, n) \frac{V_{n}^{q(1-\sigma)-1}}{v_{n}^{q-1}} a_{n}^{q}\right\}^{\frac{1}{q}} .
\end{aligned}
$$

Then by (18) we have (44).

By the reverse Hölder inequality we have

$$
\begin{aligned}
I & =\int_{0}^{\infty}\left(\frac{U^{\frac{1}{q}-\delta \sigma}(x)}{\mu^{\frac{1}{q}}(x)} f(x)\right)\left[\frac{\mu^{\frac{1}{q}}(x)}{U^{\frac{1}{q}-\delta \sigma}(x)} \sum_{n=1}^{\infty} \frac{\operatorname{sech}\left(\rho\left(U^{\delta}(x) V_{n}\right)^{\gamma}\right)}{e^{\alpha\left(U^{\delta}(x) V_{n}\right)^{\gamma}}} a_{n}\right] d x \\
& \geq\|f\|_{p, \Phi_{\delta}} J_{2} .
\end{aligned}
$$

Then by (44) we have (42). On the other hand, assuming that (44) is valid, we set $f(x)$ as in Theorem 1 . Then we find $J_{2}^{q}=\|f\|_{p, \Phi_{\delta}}^{p}$. If $J_{2}=\infty$, then (44) is trivially valid; if $J_{2}=0$, then (44) keeps impossible. Suppose that $0<J_{2}<\infty$. By (42) it follows that

$$
\|f\|_{p, \Phi_{\delta}}^{p}=J_{2}^{q}=I>k(\sigma)\|f\|_{p, \Phi_{\delta}}\|a\|_{q, \Psi}, \quad\|f\|_{p, \Phi_{\delta}}^{p-1}=J_{2}>k(\sigma)\|a\|_{q, \Psi},
$$

and then (44) follows, which is equivalent to (42).

Therefore, inequalities (42), (43), and (44) are equivalent.

For $\varepsilon \in(0, q \sigma)$, we set $\tilde{\sigma}=\sigma-\frac{\varepsilon}{q}$ and $\tilde{f}=\tilde{f}(x), x \in \mathbf{R}_{+}, \tilde{a}=\left\{\tilde{a}_{n}\right\}_{n=1}^{\infty}$,

$$
\begin{aligned}
& \tilde{f}(x)= \begin{cases}U^{\delta(\tilde{\sigma}+\varepsilon)-1}(x) \mu(x), & 0<x^{\delta} \leq 1, \\
0, & x^{\delta}>0,\end{cases} \\
& \tilde{a}_{n}=\widetilde{V}_{n}^{\tilde{\sigma}-1} v_{n}=\widetilde{V}_{n}^{\sigma-\frac{\varepsilon}{q}-1} v_{n}, \quad n \in \mathbf{N} .
\end{aligned}
$$

By (20), (32), and (16) we obtain

$$
\begin{aligned}
& \|\tilde{f}\|_{p, \Phi_{\delta}}\|\tilde{a}\|_{q, \Psi}=\frac{1}{\varepsilon} U^{\frac{\delta \varepsilon}{p}}(1)\left(\frac{1}{V_{n_{0}}^{\varepsilon}}+\varepsilon O(1)\right)^{\frac{1}{q}}, \\
& \tilde{I}=\sum_{n=1}^{\infty} \int_{0}^{\infty} \frac{\operatorname{sech}\left(\rho\left(U^{\delta}(x) V_{n}\right)^{\gamma}\right)}{e^{\alpha\left(U^{\delta}(x) V_{n}\right)^{\gamma}}} \tilde{a}_{n} \tilde{f}(x) d x
\end{aligned}
$$




$$
\begin{aligned}
& =\int_{\left\{x>0 ; 0<x^{\delta} \leq 1\right\}} \omega_{\delta}(\tilde{\sigma}, x) \frac{\mu(x)}{U^{1-\delta \varepsilon}(x)} d x \\
& \leq k(\tilde{\sigma}) \int_{\left\{x>0 ; 0<x^{\delta} \leq 1\right\}} \frac{\mu(x)}{U^{1-\delta \varepsilon}(x)} d x=\frac{1}{\varepsilon} k\left(\sigma-\frac{\varepsilon}{q}\right) U^{\delta \varepsilon}(1) .
\end{aligned}
$$

If there exists a positive constant $K \geq k(\sigma)$ such that (42) is valid when replacing $k(\sigma)$ by $K$, then, in particular, we have $\varepsilon \tilde{I}>\varepsilon K\|\tilde{f}\|_{p, \Phi_{\delta}}\|\tilde{a}\|_{q, \Psi}$, namely,

$$
k\left(\sigma-\frac{\varepsilon}{q}\right) U^{\delta \varepsilon}(1)>K \cdot U^{\frac{\delta \varepsilon}{p}}(1)\left(\frac{1}{V_{n_{0}}^{\varepsilon}}+\varepsilon O(1)\right)^{\frac{1}{q}} .
$$

It follows that $k(\sigma) \geq K\left(\varepsilon \rightarrow 0^{+}\right)$. Hence, $K=k(\sigma)$ is the best possible constant factor of (42).

The constant factor $k(\sigma)$ in (43) ((44)) is still the best possible. Otherwise, we would reach a contradiction by $(45)((46))$ that the constant factor in (42) is not the best possible.

Theorem 4 With the assumptions of Theorem 3, if $0<p<1,0<\|f\|_{p, \Phi_{\delta}}$, and $\|a\|_{q, \Psi}<\infty$, then we have the following equivalent inequalities with the best possible constant factor $k(\sigma)$ :

$$
\begin{aligned}
I & =\sum_{n=1}^{\infty} \int_{0}^{\infty} \frac{\operatorname{sech}\left(\rho\left(U^{\delta}(x) V_{n}\right)^{\gamma}\right)}{e^{\alpha\left(U^{\delta}(x) V_{n}\right)^{\gamma}}} a_{n} f(x) d x>k(\sigma)\|f\|_{p, \widetilde{\Phi}_{\delta}}\|a\|_{q, \Psi}, \\
J_{1} & =\sum_{n=1}^{\infty} \frac{v_{n}}{V_{n}^{1-p \sigma}}\left[\int_{0}^{\infty} \frac{\operatorname{sech}\left(\rho\left(U^{\delta}(x) V_{n}\right)^{\gamma}\right)}{\left.e^{\alpha\left(U^{\delta}(x) V_{n}\right)^{\gamma}} f(x) d x\right]^{p}>k(\sigma)\|f\|_{p, \widetilde{\Phi}_{\delta}},}\right. \\
J & :=\left\{\int_{0}^{\infty} \frac{\left(1-\theta_{\delta}(\sigma, x)\right)^{1-q} \mu(x)}{U^{1-q \delta \sigma}(x)}\left[\sum_{n=1}^{\infty} \frac{\operatorname{sech}\left(\rho\left(U^{\delta}(x) V_{n}\right)^{\gamma}\right)}{e^{\alpha\left(U^{\delta}(x) V_{n}\right)^{\gamma}}} a_{n}\right]^{q} d x\right\}^{\frac{1}{q}} \\
& >k(\sigma)\|a\|_{q, \Psi} .
\end{aligned}
$$

Proof By the reverse Hölder inequality with weight, since $0<p<1$, similarly as obtaining (24) and (25), we have

$$
\begin{aligned}
& {\left[\int_{0}^{\infty} \frac{\operatorname{sech}\left(\rho\left(U^{\delta}(x) V_{n}\right)^{\gamma}\right)}{\left.e^{\alpha\left(U^{\delta}(x) V_{n}\right)^{\gamma}} f(x) d x\right]^{p}}\right.} \\
& \quad \geq \frac{\left(\varpi_{\delta}(\sigma, n)\right)^{p-1}}{V_{n}^{p \sigma-1} v_{n}} \int_{0}^{\infty} \frac{\operatorname{sech}\left(\rho\left(U^{\delta}(x) V_{n}\right)^{\gamma}\right)}{e^{\alpha\left(U^{\delta}(x) V_{n}\right)^{\gamma}}} \frac{U^{(1-\delta \sigma)(p-1)}(x) v_{n}}{V_{n}^{1-\sigma} \mu^{p-1}(x)} f^{p}(x) d x .
\end{aligned}
$$

In view of (18) and the Lebesgue term-by-term integration theorem, we find

$$
\begin{aligned}
J_{1} & \geq(k(\sigma))^{\frac{1}{q}}\left[\sum_{n=1}^{\infty} \int_{0}^{\infty} \frac{\operatorname{sech}\left(\rho\left(U^{\delta}(x) V_{n}\right)^{\gamma}\right)}{e^{\alpha\left(U^{\delta}(x) V_{n}\right)^{\gamma}}} \frac{U^{(1-\delta \sigma)(p-1)}(x) v_{n}}{V_{n}^{1-\sigma} \mu^{p-1}(x)} f^{p}(x) d x\right]^{\frac{1}{p}} \\
& =(k(\sigma))^{\frac{1}{q}}\left[\int_{0}^{\infty} \omega_{\delta}(\sigma, x) \frac{U^{p(1-\delta \sigma)-1}(x)}{\mu^{p-1}(x)} f^{p}(x) d x\right]^{\frac{1}{p}} .
\end{aligned}
$$

Then by (19) we have (48). 
By the reverse Hölder inequality we have

$$
\begin{aligned}
I & =\sum_{n=1}^{\infty}\left[\frac{v_{n}^{\frac{1}{p}}}{V_{n}^{\frac{1}{p}-\sigma}} \int_{0}^{\infty} \frac{\sec h\left(\rho\left(U^{\delta}(x) V_{n}\right)^{\gamma}\right)}{e^{\alpha\left(U^{\delta}(x) V_{n}\right)^{\gamma}}} f(x) d x\right]\left(\frac{V_{n}^{\frac{1}{p}-\sigma} a_{n}}{v_{n}^{\frac{1}{p}}}\right) \\
& \geq J_{1}\|a\|_{q, \Psi .}
\end{aligned}
$$

Then by (48) we have (47). On the other hand, assuming that (47) is valid, we set $a_{n}$ as in Theorem 1 . Then we find $J_{1}^{p}=\|a\|_{q, \Psi}^{q}$. If $J_{1}=\infty$, then (48) is trivially valid; if $J_{1}=0$, then (48) keeps impossible. Suppose that $0<J_{1}<\infty$. By (47) it follows that

$$
\|a\|_{q, \Psi}^{q}=J_{1}^{p}=I>k(\sigma)\|f\|_{p, \widetilde{\Phi}_{\delta}}\|a\|_{q, \Psi}, \quad\|a\|_{q, \Psi}^{q-1}=J_{1}>k(\sigma)\|f\|_{p, \widetilde{\Phi}_{\delta}}
$$

and then (48) follows, which is equivalent to (47).

Again by the reverse Hölder inequality with weight, since $q<0$, we have

$$
\begin{aligned}
& {\left[\sum_{n=1}^{\infty} \frac{\sec h\left(\rho\left(U^{\delta}(x) V_{n}\right)^{\gamma}\right)}{e^{\alpha\left(U^{\delta}(x) V_{n}\right)^{\gamma}}} a_{n}\right]^{q}} \\
& \quad \leq \frac{\left(\omega_{\delta}(\sigma, x)\right)^{q-1}}{U^{q \delta \sigma-1}(x) \mu(x)} \sum_{n=1}^{\infty} \frac{\sec h\left(\rho\left(U^{\delta}(x) V_{n}\right)^{\gamma}\right)}{e^{\alpha\left(U^{\delta}(x) V_{n}\right)^{\gamma}}} \frac{V_{n}^{(1-\sigma)(q-1)} \mu(x)}{U^{1-\delta \sigma}(x) v_{n}^{q-1}} a_{n}^{q} .
\end{aligned}
$$

Then by (19) and the Lebesgue term-by-term integration theorem it follows that

$$
\begin{aligned}
J>(k(\sigma))^{\frac{1}{p}}\left\{\int_{0}^{\infty} \sum_{n=1}^{\infty} \frac{\operatorname{sech}\left(\rho\left(U^{\delta}(x) V_{n}\right)^{\gamma}\right)}{e^{\alpha\left(U^{\delta}(x) V_{n}\right)^{\gamma}}} \frac{V_{n}^{(1-\sigma)(q-1)} \mu(x)}{U^{1-\delta \sigma}(x) \nu_{n}^{q-1}} a_{n}^{q} d x\right\}^{\frac{1}{q}} \\
=(k(\sigma))^{\frac{1}{p}}\left\{\sum_{n=1}^{\infty} \varpi_{\delta}(\sigma, n) \frac{V_{n}^{q(1-\sigma)-1}}{v_{n}^{q-1}} a_{n}^{q}\right\}^{\frac{1}{q}} .
\end{aligned}
$$

Then by (18) we have (49).

By the reverse Hölder inequality we have

$$
\begin{aligned}
I= & \int_{0}^{\infty}\left[\left(1-\theta_{\delta}(\sigma, x)\right)^{\frac{1}{p}} \frac{U^{\frac{1}{q}-\delta \sigma}(x)}{\mu^{\frac{1}{q}}(x)} f(x)\right] \\
& \times\left[\frac{\left(1-\theta_{\delta}(\sigma, x)\right)^{\frac{-1}{p}} \mu^{\frac{1}{q}}(x)}{U^{\frac{1}{q}-\delta \sigma}(x)} \sum_{n=1}^{\infty} \frac{\sec h\left(\rho\left(U^{\delta}(x) V_{n}\right)^{\gamma}\right)}{e^{\alpha\left(U^{\delta}(x) V_{n}\right)^{\gamma}}} a_{n}\right] d x \\
\geq & \|f\|_{p, \widetilde{\Phi}_{\delta} J .}
\end{aligned}
$$

Then by (49) we have (47). On the other hand, assuming that (47) is valid, we set $f(x)$ as in Theorem 1 . Then we find $J^{q}=\|f\|_{p, \widetilde{\Phi}_{\delta}}^{p}$. If $J=\infty$, then (49) is trivially valid; if $J=0$, then (49) keeps impossible. Suppose that $0<J<\infty$. By (47) it follows that

$$
\|f\|_{p, \widetilde{\Phi}_{\delta}}^{p}=J^{q}=I>k(\sigma)\|f\|_{p, \widetilde{\Phi}_{\delta}}\|a\|_{q, \Psi}, \quad\|f\|_{p, \widetilde{\Phi}_{\delta}}^{p-1}=J>k(\sigma)\|a\|_{q, \Psi},
$$

and then (49) follows, which is equivalent to (47). 
Therefore, inequalities (47), (48), and (49) are equivalent.

For $\varepsilon \in(0, p \sigma)$, we set $\tilde{\sigma}=\sigma+\frac{\varepsilon}{p}$ and $\tilde{f}=\tilde{f}(x), x \in \mathbf{R}_{+}, \tilde{a}=\left\{\tilde{a}_{n}\right\}_{n=1}^{\infty}$,

$$
\begin{aligned}
& \tilde{f}(x)= \begin{cases}U^{\delta \tilde{\sigma}-1}(x) \mu(x), & 0<x^{\delta} \leq 1, \\
0, & x^{\delta}>0,\end{cases} \\
& \tilde{a}_{n}=\tilde{V}_{n}^{\tilde{\sigma}-\varepsilon-1} v_{n}=\widetilde{V}_{n}^{\sigma-\frac{\varepsilon}{q}-1} v_{n}, \quad n \in \mathbf{N} .
\end{aligned}
$$

By (19), (20), and (32) we obtain

$$
\begin{aligned}
& \|\tilde{f}\|_{p, \widetilde{\Phi}_{\delta}}\|\tilde{a}\|_{q, \Psi}=\left[\int_{\left\{x>0 ; 0<x^{\delta} \leq 1\right\}}\left(1-O\left((U(x))^{\delta \sigma}\right)\right) \frac{\mu(x) d x}{U^{1-\delta \varepsilon}(x)}\right]^{\frac{1}{p}}\left(\sum_{n=1}^{\infty} \frac{v_{n}}{V_{n}^{1+\varepsilon}}\right)^{\frac{1}{q}} \\
& =\frac{1}{\varepsilon}\left(U^{\delta \varepsilon}(1)-\varepsilon O(1)\right)^{\frac{1}{p}}\left(\frac{1}{V_{n_{0}}^{\varepsilon}}+\varepsilon O(1)\right)^{\frac{1}{q}}, \\
& \tilde{I}=\sum_{n=1}^{\infty} \int_{0}^{\infty} \frac{\operatorname{sech}\left(\rho\left(U^{\delta}(x) V_{n}\right)^{\gamma}\right)}{e^{\alpha\left(U^{\delta}(x) V_{n}\right)^{\gamma}}} \tilde{a}_{n} \tilde{f}(x) d x \\
& =\sum_{n=1}^{\infty}\left(\int_{\left\{x>0 ; 0<x^{\delta} \leq 1\right\}} \frac{\operatorname{sech}\left(\rho\left(U^{\delta}(x) V_{n}\right)^{\gamma}\right)}{e^{\alpha\left(U^{\delta}(x) V_{n}\right)^{\gamma}}} \frac{V_{n}^{\tilde{\sigma}} \mu(x)}{U^{1-\delta \tilde{\sigma}}(x)} d x\right) \frac{v_{n}}{V_{n}^{1+\varepsilon}} \\
& \leq \sum_{n=1}^{\infty}\left(\int_{0}^{\infty} \frac{\operatorname{sech}\left(\rho\left(U^{\delta}(x) V_{n}\right)^{\gamma}\right)}{e^{\alpha\left(U^{\delta}(x) V_{n}\right)^{\gamma}}} \frac{V_{n}^{\tilde{\sigma}} \mu(x)}{U^{1-\delta \tilde{\sigma}}(x)} d x\right) \frac{v_{n}}{V_{n}^{1+\varepsilon}} \\
& =\sum_{n=1}^{\infty} \varpi_{\delta}(\tilde{\sigma}, n) \frac{v_{n}}{V_{n}^{1+\varepsilon}}=k(\tilde{\sigma}) \sum_{n=1}^{\infty} \frac{v_{n}}{V_{n}^{1+\varepsilon}} \\
& =\frac{1}{\varepsilon} k\left(\sigma+\frac{\varepsilon}{p}\right)\left(\frac{1}{V_{n_{0}}^{\varepsilon}}+\varepsilon O(1)\right) \text {. }
\end{aligned}
$$

If there exists a positive constant $K \geq k(\sigma)$ such that (42) is valid when replacing $k(\sigma)$ by $K$, then, in particular, we have $\varepsilon \tilde{I}>\varepsilon K\|\tilde{f}\|_{p, \widetilde{\Phi}_{\delta}}\|\tilde{a}\|_{q, \Psi}$, namely,

$$
k\left(\sigma+\frac{\varepsilon}{p}\right)\left(\frac{1}{V_{n_{0}}^{\varepsilon}}+\varepsilon O(1)\right)>K\left(U^{\delta \varepsilon}(1)-\varepsilon O(1)\right)^{\frac{1}{p}}\left(\frac{1}{V_{n_{0}}^{\varepsilon}}+\varepsilon O(1)\right)^{\frac{1}{q}} .
$$

It follows that $k(\sigma) \geq K\left(\varepsilon \rightarrow 0^{+}\right)$. Hence, $K=k(\sigma)$ is the best possible constant factor of (47).

The constant factor $k(\sigma)$ in (48) ((49)) is still the best possible. Otherwise, we would reach a contradiction by $(50)((51))$ that the constant factor in $(47)$ is not the best possible.

\section{Some corollaries}

For $\delta=1$ in Theorems 2-4, we have the following inequalities with inhomogeneous kernel.

Corollary 1 If $\gamma, \rho>0, \alpha>\rho(\alpha \geq 0), 0<\sigma \leq 1, k(\sigma)$ is indicated by (12), there exists $n_{0} \in \mathbf{N}$ such that $v_{n} \geq v_{n+1}\left(n \in\left\{n_{0}, n_{0}+1, \ldots\right\}\right)$, and $U(\infty)=V(\infty)=\infty$, then 
(i) for $p>1,0<\|f\|_{p, \Phi_{1}},\|a\|_{q, \Psi}<\infty$, we have the following equivalent inequalities:

$$
\begin{aligned}
& \sum_{n=1}^{\infty} \int_{0}^{\infty} \frac{\operatorname{sech}\left(\rho\left(U(x) V_{n}\right)^{\gamma}\right)}{e^{\alpha\left(U(x) V_{n}\right)^{\gamma}}} a_{n} f(x) d x<k(\sigma)\|f\|_{p, \Phi_{1}}\|a\|_{q, \Psi}, \\
& \sum_{n=1}^{\infty} \frac{v_{n}}{V_{n}^{1-p \sigma}}\left[\int_{0}^{\infty} \frac{\operatorname{sech}\left(\rho\left(U(x) V_{n}\right)^{\gamma}\right)}{e^{\alpha\left(U(x) V_{n}\right)^{\gamma}}} f(x) d x\right]^{p}<k(\sigma)\|f\|_{p, \Phi_{1}}, \\
& \left\{\int_{0}^{\infty} \frac{\mu(x)}{U^{1-q \sigma}(x)}\left[\sum_{n=1}^{\infty} \frac{\operatorname{sech}\left(\rho\left(U(x) V_{n}\right)^{\gamma}\right)}{e^{\alpha\left(U(x) V_{n}\right)^{\gamma}}} a_{n}\right]^{q} d x\right\}^{\frac{1}{q}}<k(\sigma)\|a\|_{q, \Psi} ;
\end{aligned}
$$

(ii) for $p<0,0<\|f\|_{p, \Phi_{1}}$, and $\|a\|_{q, \Psi}<\infty$, we have the following equivalent inequalities:

$$
\begin{aligned}
& \sum_{n=1}^{\infty} \int_{0}^{\infty} \frac{\operatorname{sech}\left(\rho\left(U(x) V_{n}\right)^{\gamma}\right)}{e^{\alpha\left(U(x) V_{n}\right)^{\gamma}}} a_{n} f(x) d x>k(\sigma)\|f\|_{p, \Phi_{1}}\|a\|_{q, \Psi}, \\
& \sum_{n=1}^{\infty} \frac{v_{n}}{V_{n}^{1-p \sigma}}\left[\int_{0}^{\infty} \frac{\operatorname{sech}\left(\rho\left(U(x) V_{n}\right)^{\gamma}\right)}{e^{\alpha\left(U(x) V_{n}\right)^{\gamma}}} f(x) d x\right]^{p}>k(\sigma)\|f\|_{p, \Phi_{1}}, \\
& \left\{\int_{0}^{\infty} \frac{\mu(x)}{U^{1-q \sigma}(x)}\left[\sum_{n=1}^{\infty} \frac{\operatorname{sech}\left(\rho\left(U(x) V_{n}\right)^{\gamma}\right)}{e^{\alpha\left(U(x) V_{n}\right)^{\gamma}}} a_{n}\right]^{q} d x\right\}^{\frac{1}{q}}>k(\sigma)\|a\|_{q, \Psi} ;
\end{aligned}
$$

(iii) for $0<p<1,0<\|f\|_{p, \Phi_{1}}$, and $\|a\|_{q, \Psi}<\infty$, we have the following equivalent inequalities:

$$
\begin{aligned}
& \sum_{n=1}^{\infty} \int_{0}^{\infty} \frac{\operatorname{sech}\left(\rho\left(U(x) V_{n}\right)^{\gamma}\right)}{e^{\alpha\left(U(x) V_{n}\right)^{\gamma}}} a_{n} f(x) d x>k(\sigma)\|f\|_{p, \widetilde{\Phi}_{1}}\|a\|_{q, \Psi}, \\
& \sum_{n=1}^{\infty} \frac{v_{n}}{V_{n}^{1-p \sigma}}\left[\int_{0}^{\infty} \frac{\operatorname{sech}\left(\rho\left(U(x) V_{n}\right)^{\gamma}\right)}{e^{\alpha\left(U(x) V_{n}\right)^{\gamma}}} f(x) d x\right]^{p}>k(\sigma)\|f\|_{p, \widetilde{\Phi}_{1}},
\end{aligned}
$$

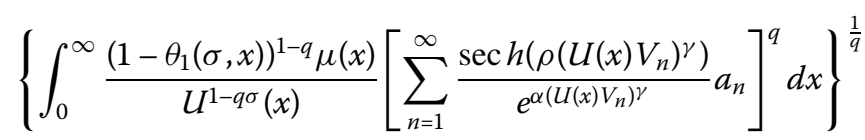

$$
\begin{aligned}
& >k(\sigma)\|a\|_{q, \Psi} .
\end{aligned}
$$

The above inequalities are with the best possible constant factor $k(\sigma)$.

For $\delta=-1$ in Theorems 2-4, we have the following inequalities with the homogeneous kernel of degree 0 .

Corollary 2 If $\gamma, \rho>0, \alpha>-\rho(\alpha \geq 0), 0<\sigma \leq 1, k(\sigma)$ is defined in (12), there exists $n_{0} \in \mathbf{N}$ such that $v_{n} \geq v_{n+1}\left(n \in\left\{n_{0}, n_{0}+1, \ldots\right\}\right)$, and $U(\infty)=V(\infty)=\infty$, then

(i) for $p>1,0<\|f\|_{p, \Phi_{-1}}$, and $\|a\|_{q, \Psi}<\infty$, we have the following equivalent inequalities:

$$
\begin{aligned}
& \sum_{n=1}^{\infty} \int_{0}^{\infty} \frac{\operatorname{sech}\left(\rho\left(\frac{V_{n}}{U(x)}\right)^{\gamma}\right)}{e^{\alpha\left(\frac{V_{n}}{U(x)}\right)^{\gamma}}} a_{n} f(x) d x<k(\sigma)\|f\|_{p, \Phi_{-1}}\|a\|_{q, \Psi}, \\
& \sum_{n=1}^{\infty} \frac{v_{n}}{V_{n}^{1-p \sigma}}\left[\int_{0}^{\infty} \frac{\sec h\left(\rho\left(\frac{V_{n}}{U(x)}\right)^{\gamma}\right)}{e^{\alpha\left(\frac{V_{n}}{U(x)}\right)^{\gamma}}} f(x) d x\right]^{p}<k(\sigma)\|f\|_{p, \Phi_{-1}},
\end{aligned}
$$




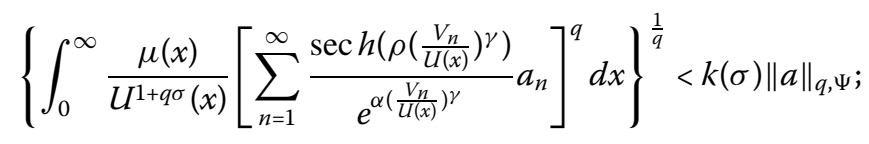

(ii) for $p<0,0<\|f\|_{p, \Phi_{-1}}$, and $\|a\|_{q, \Psi}<\infty$, we have the following equivalent inequalities:

$$
\begin{aligned}
& \sum_{n=1}^{\infty} \int_{0}^{\infty} \frac{\operatorname{sech}\left(\rho\left(\frac{V_{n}}{U(x)}\right)^{\gamma}\right)}{e^{\alpha\left(\frac{V_{n}}{U(x)}\right)^{\gamma}}} a_{n} f(x) d x>k(\sigma)\|f\|_{p, \Phi_{-1}}\|a\|_{q, \Psi}, \\
& \sum_{n=1}^{\infty} \frac{v_{n}}{V_{n}^{1-p \sigma}}\left[\int_{0}^{\infty} \frac{\operatorname{sech}\left(\rho\left(\frac{V_{n}}{U(x)}\right)^{\gamma}\right)}{\left.e^{\alpha\left(\frac{V_{n}}{U(x)}\right)^{\gamma}} f(x) d x\right]^{p}>k(\sigma)\|f\|_{p, \Phi_{-1}},}\right. \\
& \left\{\int_{0}^{\infty} \frac{\mu(x)}{U^{1+q \sigma}(x)}\left[\sum_{n=1}^{\infty} \frac{\operatorname{sech}\left(\rho\left(\frac{V_{n}}{U(x)}\right)^{\gamma}\right)}{e^{\alpha\left(\frac{V_{n}}{U(x)}\right)^{\gamma}}} a_{n}\right]^{q} d x\right\}^{\frac{1}{q}}>k(\sigma)\|a\|_{q, \Psi} ;
\end{aligned}
$$

(iii) for $0<p<1,0<\|f\|_{p, \Phi_{-1}}$, and $\|a\|_{q, \Psi}<\infty$, we have the following equivalent inequalities:

$$
\begin{aligned}
& \sum_{n=1}^{\infty} \int_{0}^{\infty} \frac{\operatorname{sech}\left(\rho\left(\frac{V_{n}}{U(x)}\right)^{\gamma}\right)}{e^{\alpha\left(\frac{V_{n}}{U(x)}\right)^{\gamma}}} a_{n} f(x) d x>k(\sigma)\|f\|_{p, \widetilde{\Phi}_{-1}}\|a\|_{q, \Psi}, \\
& \sum_{n=1}^{\infty} \frac{v_{n}}{V_{n}^{1-p \sigma}}\left[\int_{0}^{\infty} \frac{\operatorname{sech}\left(\rho\left(\frac{V_{n}}{U(x)}\right)^{\gamma}\right)}{e^{\alpha\left(\frac{V_{n}}{U(x)}\right)^{\gamma}}} f(x) d x\right]^{p}>k(\sigma)\|f\|_{p, \widetilde{\Phi}_{-1}},
\end{aligned}
$$

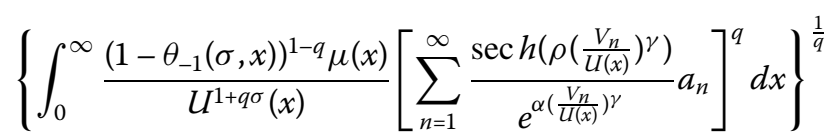

$$
\begin{aligned}
& >k(\sigma)\|a\|_{q, \Psi} .
\end{aligned}
$$

The above inequalities are with the best possible constant factor $k(\sigma)$.

For $\alpha=\rho$ and $\gamma=\sigma$ in Theorems 2-4, we have the following corollary.

Corollary 3 If $\rho>0,0<\sigma \leq 1$, there exists $n_{0} \in \mathbf{N}$ such that $v_{n} \geq v_{n+1}\left(n \in\left\{n_{0}, n_{0}+1, \ldots\right\}\right)$, and $U(\infty)=V(\infty)=\infty$, then

(i) for $p>1,0<\|f\|_{p, \Phi_{\delta}},\|a\|_{q, \Psi}<\infty$, we have the following equivalent inequalities with the best possible constant factor $\frac{\ln 2}{\sigma \rho}$ :

$$
\begin{aligned}
& \sum_{n=1}^{\infty} \int_{0}^{\infty} \frac{\operatorname{sech}\left(\rho\left(U^{\delta}(x) V_{n}\right)^{\sigma}\right)}{e^{\rho\left(U^{\delta}(x) V_{n}\right)^{\sigma}}} a_{n} f(x) d x<\frac{\ln 2}{\sigma \rho}\|f\|_{p, \Phi_{\delta}}\|a\|_{q, \Psi}, \\
& \sum_{n=1}^{\infty} \frac{v_{n}}{V_{n}^{1-p \sigma}}\left[\int_{0}^{\infty} \frac{\operatorname{sech}\left(\rho\left(U^{\delta}(x) V_{n}\right)^{\sigma}\right)}{e^{\rho\left(U^{\delta}(x) V_{n}\right)^{\sigma}}} f(x) d x\right]^{p}<\frac{\ln 2}{\sigma \rho}\|f\|_{p, \Phi_{\delta}} \\
& \left\{\int_{0}^{\infty} \frac{\mu(x)}{U^{1-q \delta \sigma}(x)}\left[\sum_{n=1}^{\infty} \frac{\operatorname{sech}\left(\rho\left(U^{\delta}(x) V_{n}\right)^{\sigma}\right)}{e^{\rho\left(U^{\delta}(x) V_{n}\right)^{\sigma}}} a_{n}\right]^{q} d x\right\}^{\frac{1}{q}} \\
& <\frac{\ln 2}{\sigma \rho}\|a\|_{q, \Psi} ;
\end{aligned}
$$


(ii) for $p<0,0<\|f\|_{p, \Phi_{\delta}},\|a\|_{q, \Psi}<\infty$, we have the following equivalent inequalities with the best possible constant factor $\frac{\ln 2}{\sigma \rho}$ :

$$
\begin{aligned}
& \sum_{n=1}^{\infty} \int_{0}^{\infty} \frac{\operatorname{sech}\left(\rho\left(U^{\delta}(x) V_{n}\right)^{\sigma}\right)}{e^{\rho\left(U^{\delta}(x) V_{n}\right)^{\sigma}}} a_{n} f(x) d x>\frac{\ln 2}{\sigma \rho}\|f\|_{p, \Phi_{\delta}}\|a\|_{q, \Psi}, \\
& \sum_{n=1}^{\infty} \frac{v_{n}}{V_{n}^{1-p \sigma}}\left[\int_{0}^{\infty} \frac{\operatorname{sech}\left(\rho\left(U^{\delta}(x) V_{n}\right)^{\sigma}\right)}{e^{\rho\left(U^{\delta}(x) V_{n}\right)^{\sigma}}} f(x) d x\right]^{p}>\frac{\ln 2}{\sigma \rho}\|f\|_{p, \Phi_{\delta}}, \\
& \left\{\int_{0}^{\infty} \frac{\mu(x)}{U^{1-q \delta \sigma}(x)}\left[\sum_{n=1}^{\infty} \frac{\operatorname{sech}\left(\rho\left(U^{\delta}(x) V_{n}\right)^{\sigma}\right)}{e^{\rho\left(U^{\delta}(x) V_{n}\right)^{\sigma}}} a_{n}\right]^{q} d x\right\}^{\frac{1}{q}}>\frac{\ln 2}{\sigma \rho}\|a\|_{q, \Psi} ;
\end{aligned}
$$

(iii) for $0<p<1,0<\|f\|_{p, \Phi_{\delta}}$, and $\|a\|_{q, \Psi}<\infty$, we have the following equivalent inequalities with the best possible constant factor $\frac{\ln 2}{\sigma \rho}$ :

$$
\begin{aligned}
& \sum_{n=1}^{\infty} \int_{0}^{\infty} \frac{\operatorname{sech}\left(\rho\left(U^{\delta}(x) V_{n}\right)^{\sigma}\right)}{e^{\rho\left(U^{\delta}(x) V_{n}\right)^{\sigma}}} a_{n} f(x) d x>\frac{\ln 2}{\sigma \rho}\|f\|_{p, \widetilde{\Phi}_{\delta}}\|a\|_{q, \Psi}, \\
& \sum_{n=1}^{\infty} \frac{v_{n}}{V_{n}^{1-p \sigma}}\left[\int_{0}^{\infty} \frac{\sec h\left(\rho\left(U^{\delta}(x) V_{n}\right)^{\sigma}\right)}{e^{\rho\left(U^{\delta}(x) V_{n}\right)^{\sigma}}} f(x) d x\right]^{p}>\frac{\ln 2}{\sigma \rho}\|f\|_{p, \widetilde{\Phi}_{\delta}} \\
& \left\{\int_{0}^{\infty} \frac{\left(1-\theta_{\delta}(\sigma, x)\right)^{1-q} \mu(x)}{U^{1-q \delta \sigma}(x)}\left[\sum_{n=1}^{\infty} \frac{\operatorname{sech}\left(\rho\left(U^{\delta}(x) V_{n}\right)^{\sigma}\right)}{e^{\rho\left(U^{\delta}(x) V_{n}\right)^{\sigma}}} a_{n}\right]^{q} d x\right\}^{\frac{1}{q}} \\
& >\frac{\ln 2}{\sigma \rho}\|a\|_{q, \Psi} .
\end{aligned}
$$

For $\alpha=0$ and $\gamma=\sigma$ in Theorems 2-4, we have the following corollary.

Corollary 4 If $\rho>0,0<\sigma \leq 1$, there exists $n_{0} \in \mathbf{N}$ such that $v_{n} \geq v_{n+1}\left(n \in\left\{n_{0}, n_{0}+1, \ldots\right\}\right)$, and $U(\infty)=V(\infty)=\infty$, then

(i) for $p>1,0<\|f\|_{p, \Phi_{\delta}}$, and $\|a\|_{q, \Psi}<\infty$, we have the following equivalent inequalities with the best possible constant factor $\frac{\pi}{2 \sigma \rho}$ :

$$
\begin{aligned}
& \sum_{n=1}^{\infty} \int_{0}^{\infty} \operatorname{sech}\left(\rho\left(U^{\delta}(x) V_{n}\right)^{\sigma}\right) a_{n} f(x) d x<\frac{\pi}{2 \sigma \rho}\|f\|_{p, \Phi_{\delta}}\|a\|_{q, \Psi}, \\
& \sum_{n=1}^{\infty} \frac{v_{n}}{V_{n}^{1-p \sigma}}\left[\int_{0}^{\infty} \operatorname{sech}\left(\rho\left(U^{\delta}(x) V_{n}\right)^{\sigma}\right) f(x) d x\right]^{p}<\frac{\pi}{2 \sigma \rho}\|f\|_{p, \Phi_{\delta}}, \\
& \left\{\int_{0}^{\infty} \frac{\mu(x)}{U^{1-q \delta \sigma}(x)}\left[\sum_{n=1}^{\infty} \operatorname{sech}\left(\rho\left(U^{\delta}(x) V_{n}\right)^{\sigma}\right) a_{n}\right]^{q} d x\right\}^{\frac{1}{q}}<\frac{\pi}{2 \sigma \rho}\|a\|_{q, \Psi} ;
\end{aligned}
$$

(ii) for $p<0,0<\|f\|_{p, \Phi_{\delta}}$, and $\|a\|_{q, \Psi}<\infty$, we have the following equivalent inequalities with the best possible constant factor $\frac{\pi}{2 \sigma \rho}$ :

$$
\sum_{n=1}^{\infty} \int_{0}^{\infty} \operatorname{sech}\left(\rho\left(U^{\delta}(x) V_{n}\right)^{\sigma}\right) a_{n} f(x) d x>\frac{\pi}{2 \sigma \rho}\|f\|_{p, \Phi_{\delta}}\|a\|_{q, \Psi}
$$




$$
\begin{aligned}
& \sum_{n=1}^{\infty} \frac{v_{n}}{V_{n}^{1-p \sigma}}\left[\int_{0}^{\infty} \operatorname{sech}\left(\rho\left(U^{\delta}(x) V_{n}\right)^{\sigma}\right) f(x) d x\right]^{p}>\frac{\pi}{2 \sigma \rho}\|f\|_{p, \Phi_{\delta}} \\
& \left\{\int_{0}^{\infty} \frac{\mu(x)}{U^{1-q \delta \sigma}(x)}\left[\sum_{n=1}^{\infty} \operatorname{sech}\left(\rho\left(U^{\delta}(x) V_{n}\right)^{\sigma}\right) a_{n}\right]^{q} d x\right\}^{\frac{1}{q}}>\frac{\pi}{2 \sigma \rho}\|a\|_{q, \Psi}
\end{aligned}
$$

(iii) for $0<p<1,0<\|f\|_{p, \Phi_{\delta}}$, and $\|a\|_{q, \Psi}<\infty$, we have the following equivalent inequalities with the best possible constant factor $\frac{\pi}{2 \sigma \rho}$ :

$$
\begin{aligned}
& \sum_{n=1}^{\infty} \int_{0}^{\infty} \operatorname{sech}\left(\rho\left(U^{\delta}(x) V_{n}\right)^{\sigma}\right) a_{n} f(x) d x>\frac{\pi}{2 \sigma \rho}\|f\|_{p, \widetilde{\Phi}_{\delta}}\|a\|_{q, \Psi}, \\
& \sum_{n=1}^{\infty} \frac{v_{n}}{V_{n}^{1-p \sigma}}\left[\int_{0}^{\infty} \operatorname{sech}\left(\rho\left(U^{\delta}(x) V_{n}\right)^{\sigma}\right) f(x) d x\right]^{p}>\frac{\pi}{2 \sigma \rho}\|f\|_{p, \widetilde{\Phi}_{\delta}}, \\
& \left\{\int_{0}^{\infty} \frac{\left(1-\theta_{\delta}(\sigma, x)\right)^{1-q} \mu(x)}{U^{1-q \delta \sigma}(x)}\left[\sum_{n=1}^{\infty} \operatorname{sech}\left(\rho\left(U^{\delta}(x) V_{n}\right)^{\sigma}\right) a_{n}\right]^{q} d x\right\}^{\frac{1}{q}} \\
& >\frac{\pi}{2 \sigma \rho}\|a\|_{q, \Psi} \text {. }
\end{aligned}
$$

Remark 2 For $\mu(x)=v_{n}=1$ in (52), we have the following inequality with the best possible constant factor $k(\sigma)$ :

$$
\begin{aligned}
& \sum_{n=1}^{\infty} \int_{0}^{\infty} \frac{\operatorname{sech}\left(\rho\left(x^{\delta} n\right)^{\gamma}\right)}{e^{\alpha\left(x^{\delta} n\right)^{\gamma}}} a_{n} f(x) d x \\
& \quad<k(\sigma)\left[\int_{0}^{\infty} x^{p(1-\delta \sigma)-1} f^{p}(x) d x\right]^{\frac{1}{p}}\left[\sum_{n=1}^{\infty} n^{q(1-\sigma)-1} a_{n}^{q}\right]^{\frac{1}{q}} .
\end{aligned}
$$

In particular, for $\delta=1$, we have the following inequality with inhomogeneous kernel:

$$
\begin{aligned}
& \sum_{n=1}^{\infty} \int_{0}^{\infty} \frac{\operatorname{sech}\left(\rho(x n)^{\gamma}\right)}{e^{\alpha(x n)^{\gamma}}} a_{n} f(x) d x \\
& \quad<k(\sigma)\left[\int_{0}^{\infty} x^{p(1-\sigma)-1} f^{p}(x) d x\right]^{\frac{1}{p}}\left[\sum_{n=1}^{\infty} n^{q(1-\sigma)-1} a_{n}^{q}\right]^{\frac{1}{q}} ;
\end{aligned}
$$

for $\delta=-1$, we have the following inequality with inhomogeneous kernel:

$$
\begin{aligned}
& \sum_{n=1}^{\infty} \int_{0}^{\infty} \frac{\operatorname{sech}\left(\rho\left(\frac{n}{x}\right)^{\gamma}\right)}{e^{\alpha\left(\frac{n}{x}\right)^{\gamma}}} a_{n} f(x) d x \\
& \quad<k(\sigma)\left[\int_{0}^{\infty} x^{p(1+\sigma)-1} f^{p}(x) d x\right]^{\frac{1}{p}}\left[\sum_{n=1}^{\infty} n^{q(1-\sigma)-1} a_{n}^{q}\right]^{\frac{1}{q}} .
\end{aligned}
$$

We still can obtain a large number of other inequalities by using some particular parameters in theorems and corollaries. 


\section{Competing interests}

The authors declare that they have no competing interests.

\section{Authors' contributions}

BY carried out the mathematical studies, participated in the sequence alignment and drafted the manuscript. QC participated in the design of the study and performed the numerical analysis. All authors read and approved the final manuscript.

\section{Author details}

'Department of Mathematics, Guangdong University of Education, Guangzhou, Guangdong 51003, P.R. China.

${ }^{2}$ Department of Computer Science, Guangdong University of Education, Guangzhou, Guangdong 51003, P.R. China.

\section{Acknowledgements}

This work is supported by the National Natural Science Foundation of China (No. 61370186) and 2013 Knowledge Construction Special Foundation Item of Guangdong Institution of Higher Learning College and University (No. 2013KJCX0140).

\section{Received: 2 July 2015 Accepted: 2 December 2015 Published online: 18 December 2015}

\section{References}

1. Hardy, GH, Littlewood, JE, Pólya, G: Inequalities. Cambridge University Press, Cambridge (1934)

2. Mitrinović, DS, Pečarić, JE, Fink, AM: Inequalities Involving Functions and Their Integrals and Derivatives. Kluwer Academic, Boston (1991)

3. Yang, BC: Hilbert-Type Integral Inequalities. Bentham Science Publishers, Sharjah (2009)

4. Yang, BC: Discrete Hilbert-Type Inequalities. Bentham Science Publishers, Sharjah (2011)

5. Yang, BC: The Norm of Operator and Hilbert-Type Inequalities. Science Press, Beijing (2009)

6. Yang, BC: On Hilbert's integral inequality. J. Math. Anal. Appl. 220, 778-785 (1998)

7. Yang, BC: An extension of a Hardy-Hilbert-type inequality. J. Guangdong Univ. Educ. 35(3), 1-8 (2015)

8. Yang, BC, Brnetić, I, Krnić, M, Pečarić, JE: Generalization of Hilbert and Hardy-Hilbert integral inequalities. Math. Inequal. Appl. 8(2), 259-272 (2005)

9. Krnić, M, Pečarić, JE: Hilbert's inequalities and their reverses. Publ. Math. (Debr.) 67(3-4), 315-331 (2005)

10. Yang, BC, Rassias, TM: On the way of weight coefficient and research for Hilbert-type inequalities. Math. Inequal. Appl. 6(4), 625-658 (2003)

11. Yang, BC, Rassias, TM: On a Hilbert-type integral inequality in the subinterval and its operator expression. Banach J. Math. Anal. 4(2), 100-110 (2010)

12. Azar, L: On some extensions of Hardy-Hilbert's inequality and applications. J. Inequal. Appl. 2009, Article ID 546829 (2009)

13. Arpad, B, Choonghong, O: Best constant for certain multilinear integral operator. J. Inequal. Appl. 2006, Article ID $28582(2006)$

14. Kuang, JC, Debnath, L: On Hilbert's type inequalities on the weighted Orlicz spaces. Pac. J. Appl. Math. 1(1), 95-103 (2007)

15. Zhong, WY: The Hilbert-type integral inequality with a homogeneous kernel of -lambda-degree. J. Inequal. Appl. 2008, Article ID 917392 (2008)

16. Hong, Y: On Hardy-Hilbert integral inequalities with some parameters. J. Inequal. Pure Appl. Math. 6(4), Article 92 (2005)

17. Zhong, WY, Yang, BC: On multiple Hardy-Hilbert's integral inequality with kernel. J. Inequal. Appl. 2007, Article ID 27962 (2007)

18. Yang, BC, Krnić, M: On the norm of a multi-dimensional Hilbert-type operator. Sarajevo J. Math. 7(20), 223-243 (2011)

19. Krnić, M, Pečarić, JE, Vuković, P: On some higher-dimensional Hilbert's and Hardy-Hilbert's type integral inequalities with parameters. Math. Inequal. Appl. 11, 701-716 (2008)

20. Krnić, M, Vuković, P: On a multidimensional version of the Hilbert-type inequality. Anal. Math. 38, 291-303 (2012)

21. Rassias, MT, Yang, BC: On half-discrete Hilbert's inequality. Appl. Math. Comput. 220, 75-93 (2013)

22. Rassias, MT, Yang, BC: A multidimensional half-discrete Hilbert-type inequality and the Riemann zeta function. Appl. Math. Comput. 225, 263-277 (2013)

23. Rassias, MT, Yang, BC: On a multidimensional half-discrete Hilbert-type inequality related to the hyperbolic cotangent function. Appl. Math. Comput. 242, 800-813 (2014)

24. Rassias, MT, Yang, BC: On a multidimensional Hilbert-type integral inequality associated to the gamma function. Appl. Math. Comput. 249, 408-418 (2014)

25. Yang, BC: On a more accurate multidimensional Hilbert-type inequality with parameters. Math. Inequal. Appl. 18(2), 429-441 (2015)

26. Yang, BC: A mixed Hilbert-type inequality with a best constant factor. Int. J. Pure Appl. Math. 20(3), 319-328 (2005)

27. Yang, BC: A half-discrete Hilbert-type inequality. J. Guangdong Univ. Educ. 31(3), 1-7 (2011)

28. Zhong, WY: A mixed Hilbert-type inequality and its equivalent forms. J. Guangdong Univ. Educ. 31(5), 18-22 (2011)

29. Zhong, WY: A half discrete Hilbert-type inequality and its equivalent forms. J. Guangdong Univ. Educ. 32(5), 8-12 (2012)

30. Zhong, JH, Yang, BC: On an extension of a more accurate Hilbert-type inequality. J. Zhejiang Univ. Sci. Ed. 35(2), 121-124 (2008)

31. Zhong, JH: Two classes of half-discrete reverse Hilbert-type inequalities with a non-homogeneous kernel. J. Guangdong Univ. Educ. 32(5), 11-20 (2012)

32. Zhong, WY, Yang, BC: A best extension of Hilbert inequality involving several parameters. J. Jinan Univ. Nat. Sci. 28(1), 20-23 (2007)

33. Zhong, WY, Yang, BC: A reverse Hilbert's type integral inequality with some parameters and the equivalent forms. Pure Appl. Math. 24(2), 401-407 (2008) 
34. Yang, BC, Chen, Q: A half-discrete Hilbert-type inequality with a homogeneous kernel and an extension. J. Inequal. Appl. 2011, 124 (2011)

35. Yang, BC: A half-discrete Hilbert-type inequality with a non-homogeneous kernel and two variables. Mediterr. J. Math. 10, 677-692 (2013)

36. Yang, BC: Two Types of Multiple Half-Discrete Hilbert-Type Inequalities. Lambert Academic Publishing, Saarbrücken (2012)

37. Yang, BC: Topics on Hlf-Discrete Hilbert-Type Inequalities. Lambert Academic Publishing, Saarbrücken (2013)

38. Yang, BC, Debnath, L: Half-Discrete Hilbert-Type Inequalities. World Scientific, Singapore (2014)

39. Zhong, YQ: Introduction to Complex Functions. Higher Education Press, Beijing (2003)

40. Wang, ZQ, Guo, DR: Introduction to Special Functions. Science Press, Beijing (1979)

41. Kuang, JC: Applied Inequalities. Shandong Science Technic Press, Jinan (2004)

42. Kuang, JC: Real Analysis and Functional Analysis. Higher Education Press, Beijing (2014)

Submit your manuscript to a SpringerOpen ${ }^{\odot}$ journal and benefit from:

- Convenient online submission

Rigorous peer review

- Immediate publication on acceptance

- Open access: articles freely available online

- High visibility within the field

- Retaining the copyright to your article 